\title{
THE CLASSIFICATION OF $p$-DIVISIBLE GROUPS OVER 2-ADIC DISCRETE VALUATION RINGS
}

\author{
WANSU KIM
}

\begin{abstract}
Let $\mathscr{O}_{K}$ be a 2 -adic discrete valuation ring with perfect residue field $k$. We classify $p$-divisible groups and $p$-power order finite flat group schemes over $\mathscr{O}_{K}$ in terms of certain Frobenius modules over $\mathfrak{S}:=W(k)[[u]]$. We also show the compatibility with crystalline Dieudonné theory and associated Galois representations. Our approach differs from Lau's generalization of display theory, who independently obtained our result using display theory.
\end{abstract}

\section{Contents}

1. Introduction

2. Review: Kisin theory

3. Review: Breuil modules

4. Statement of the main result

5. Proof of the main results

\section{Introduction}

Let $k$ be a perfect field of characteristic $p, W(k)$ its ring of Witt vectors, and $K_{0}:=$ $W(k)\left[\frac{1}{p}\right]$. Let $K$ be a finite totally ramified extension of $K_{0}$ and let us fix its algebraic closure $\bar{K}$. We fix a uniformizer $\pi \in K$ and an Eisenstein polynomial $E(u) \in W(k)[u]$ such that $E(\pi)=0$ and $E(0)=p$. Choose $\pi^{(n)} \in \bar{K}$ so that $\left(\pi^{(n+1)}\right)^{p}=\pi^{(n)}$ and $\pi^{(0)}=\pi$. Put $K_{\infty}:=\bigcup_{n} K\left(\pi^{(n)}\right), \mathcal{G}_{K}:=\operatorname{Gal}(\bar{K} / K)$ and $\mathcal{G}_{K_{\infty}}:=\operatorname{Gal}\left(\bar{K} / K_{\infty}\right)$.

Set $\mathfrak{S}:=W(k)[[u]]$ and we extend the Witt vector Frobenius map to a ring endomorphism $\varphi: \mathfrak{S} \rightarrow \mathfrak{S}$ by $\varphi(u)=u^{p}$. Breuil has conjectured that $p$-divisible groups and finite flat group schemes over $\mathscr{O}_{K}$ are classified by a certain Frobenius-module over $\mathfrak{S}$; namely, $\mathrm{BT}_{/ \mathfrak{S}}^{\varphi}$ and $(\operatorname{Mod} / \mathfrak{S})^{\leqslant 1}$, respectively. (The precise definitions will be given later in Definitions 2.1 and 2.5.) Also note that there exist contravariant functors $T_{\mathfrak{S}}^{*}$ from $\mathrm{BT}_{/ \mathfrak{S}}^{\varphi}$ and $(\operatorname{Mod} / \mathfrak{S})^{\leqslant 1}$ to the category of finitely generated $\mathbb{Z}_{p}$-modules with continuous $\mathcal{G}_{K_{\infty}}$-action (equations (2.2.1) and (2.6.2)). The main result of this paper is the proof of the following conjecture of Breuil.

Received by the editors May 4, 2011.

2000 Mathematics Subject Classification. 11S20, 14F30.

Key words and phrases. classification of finite flat group schemes, Kisin theory. 
Conjecture 1.1 (Breuil). There exists an exact anti-equivalence of categories $G \rightsquigarrow$ $\mathfrak{M}_{G}$ between the category of $p$-divisible groups over $\mathscr{O}_{K}$ and $\mathrm{BT}_{/ \mathfrak{S}}^{\varphi}$ such that the contravariant Dieudonné crystal $\mathbb{D}^{*}(G)$ can be recovered from $\mathfrak{M}_{G}$ and there exists a natural $\mathcal{G}_{K_{\infty}}$-equivariant isomorphism $T_{p}(G) \cong T_{\mathfrak{S}}^{*}\left(\mathfrak{M}_{G}\right)$.

There exists an exact anti-equivalence of categories $H \leadsto \mathfrak{M}_{H}$ between the category of p-power order finite flat group schemes over $\mathscr{O}_{K}$ and $(\operatorname{Mod} / \mathfrak{S})^{\leqslant 1}$ such that the contravariant Dieudonné crystal $\mathbb{D}^{*}(H)$ can be recovered from $\mathfrak{M}_{H}$ and there exists a natural $\mathcal{G}_{K_{\infty}}$-equivariant isomorphism $H(\bar{K}) \cong T_{\mathfrak{S}}^{*}\left(\mathfrak{M}_{H}\right)$.

The definition of these functors make use of the choice of uniformizer $\pi \in \mathscr{O}_{K}$ (since the definitions of the target categories $\mathrm{BT}_{/ \mathfrak{S}}^{\varphi}$ and $\left.(\operatorname{Mod} / \mathfrak{S})^{\leqslant 1} \mathrm{do}\right) .{ }^{1}$ Note that the $\mathcal{G}_{K_{\infty}}$-restriction induces a fully faithful functor from the category of $\mathcal{G}_{K^{-}}$ representations coming from either a $p$-divisible group or a finite flat group scheme over $\mathscr{O}_{K}$ to the category of $\mathcal{G}_{K_{\infty}}$-representations (cf. [12, Corollary 2.1.14] and Corollary 4.4 in this paper), and when $G$ is a $p$-divisible group we can give a description of the $\mathcal{G}_{K}$-action on $T_{\mathfrak{S}}^{*}\left(\mathfrak{M}_{G}\right)$, which recovers the natural $\mathcal{G}_{K}$-action on $T_{p}(G)$. (cf. equations (3.5.2a) and (3.5.2b), Proposition 5.4, and the proof of Proposition 5.5.)

Although the compatibility of the functors with the associated Galois representations was not explicitly stated in the original conjecture [5, Section 2], it is important for the applications in number theory; for example, this is needed for the construction of moduli of finite flat group schemes [14], and Kisin's strategy of constructing a good integral model of an abelian-type Shimura variety $[15] .{ }^{2}$ In the latter case we need this extra compatibility because Kisin's argument requires that the assignment $T_{p}(G) \rightsquigarrow \mathfrak{M}_{G}$ for a $p$-divisible group $G$ over $\mathscr{O}_{K}$ should extend to a $\otimes$-functor on the $\otimes$-category generated by the $T_{p}(G)$ 's in the category of $\mathbb{Z}_{p}\left[\mathcal{G}_{K}\right]$-modules (hence, we escape the realm of Barsotti-Tate representations), and this could be checked via Galois compatibility.

Now we comment on the previous results on the conjecture. Breuil $[5,6]$ proved the conjecture for finite flat group schemes killed by $p$ when $p>2$. Kisin $[12$, Section A and Corollary 2.2.6] proved the full conjecture when $p>2$, and proved the "isogeny version" of the conjecture when $p=2$. Later Kisin [13, Section 1] proved the conjecture for the Cartier duals of connected $p$-divisible groups without restriction on $p$, using Zink's theory of windows and displays. In this paper, we prove the conjecture without connectedness assumptions when $p=2$.

Independently, Eike Lau $[19,18]$ has proven the conjecture by a different approach. Indeed, Lau [19] extended the Zink's theory of windows and displays for arbitrary $p$-divisible groups with no restrictions on $p$ (over more general bases than discrete valuation rings), and obtained the compatibility with Galois representations in [18] via "displayed equation". Shortly after this paper was completed, Tong Liu [22] independently proved Theorem 4.1 of this paper using his theory of $(\varphi, \hat{G})$-modules. Our approach is different from aforementioned results, and more related to Kisin's original approach in [12].

\footnotetext{
${ }^{1}$ Note that for fixed $\pi=\pi^{(0)}$ any two choices of $\mathcal{G}_{K_{\infty}} \subset \mathcal{G}_{K}$ are conjugate to each other.

${ }^{2}$ Note that Vasiu [27] has claimed to have proved the main result of [15] including 2-adic case without using Conjecture 1.1.
} 
The $p=2$ case of Conjecture 1.1 has been the main technical obstacle to constructing a good 2-adic integral model of a Shimura variety of abelian type following Kisin's strategy [15]. Also Madapusi Pera's work on toroidal compactifications of these integral models in the Hodge-type case [23] assumes $p>2$ for the similar reason. Although we have not succeeded yet, it seems that the result of this paper (which proves Conjecture 1.1) can be used to extend the main results of [15] (and quite possibly, [23]) to cover some 2-adic cases. (See Remark 4.6 for more details.) Note that the existence of such good integral models plays a fundamental role in Langlands' Jugendtraum [17] and Kudla's program outlined in [16].

Now, let us explain some of the trouble one encounters when proving Conjecture 1.1 when $p=2$ (with no connectedness assumptions). We consider the following the $\mathbb{Z}_{p^{-}}$ lattices in $V_{p}(G):=T_{p}(G)\left[\frac{1}{p}\right]$ for a $p$-divisible group $G$ over $\mathscr{O}_{K}$.

- $T:=T_{p}(G)$, the $p$-adic Tate module.

- $T_{\mathfrak{M}}:=T_{\mathfrak{S}}^{*}\left(\mathfrak{M}_{G}\right)$, where $T_{\mathfrak{S}}^{*}\left(\mathfrak{M}_{G}\right)$ is defined in equation $(2.2 .1)$.

- $T_{\mathbb{D}}$, the $\mathbb{Z}_{p}$-lattice associated to the Dieudonné crystal via $T_{\mathrm{st}}^{*}$ (equations (3.5.1a) and (3.5.1b)). cf. Section 5.6.

The desired compatibility follows from the equality $T=T_{\mathfrak{M}}$.

When $p>2$ or when the Cartier dual $G^{\vee}$ is connected, we can prove the equality $T=T_{\mathfrak{M}}$ by showing $T=T_{\mathbb{D}}$ and $T_{\mathfrak{M}}=T_{\mathbb{D}}$. Indeed, the equality $T_{\mathfrak{M}}=T_{\mathbb{D}}$ follows from a standard linear algebra computation together with the compatibility with crystalline Dieudonné theory, if $p>2$ or if the Cartier dual $G^{\vee}$ is connected. The equality $T=T_{\mathbb{D}}$ follows from Faltings's integral comparison isomorphism when $p>2$ $\left[9\right.$, Theorem 7] and Zink's theory of windows and display when $G^{\vee}$ is connected $[13$, Proposition 1.1.10]. The trouble is that when $p=2$ the lattice $T_{\mathfrak{M}}$ is strictly contained in $T_{\mathbb{D}}$ if $G^{\vee}$ is not connected (Proposition 5.4). Indeed, we will show that $T=T_{\mathfrak{M}}$ as sublattices in $T_{\mathbb{D}}$.

Let us explain our approach to prove Conjecture 1.1. We use the Kisin theory [12] to define a functor $G \rightsquigarrow \mathfrak{M}_{G}$ from the category of $p$-divisible groups to the category of $(\varphi, \mathfrak{S})$-modules in such a way that the equality $T=T_{\mathfrak{M}}$ is automatic by construction. We show that this functor is an anti-equivalence, and then we separately show the compatibility with Dieudonné crystals. Since the result up to isogeny was already obtained by Kisin [12], it is not surprising that the integral results can be obtained by studying Galois-stable lattices in $V_{p}(G)$.

The author expects that this idea can be generalized to show the following statement about $\mathcal{G}_{K}$-stable $\mathbb{Z}_{p}$-lattices in semi-stable representations with small HodgeTate weights.

Conjecture 1.2. Let $D:=\left(D, \varphi_{D}, N, \mathrm{Fil}^{\bullet} D_{K}\right)$ be a weakly admissible filtered $(\varphi, N)$ module such that $\operatorname{gr}^{w} D_{K}=0$ for all $w \notin[0, p-1]$. Consider $\mathfrak{M} \in \operatorname{Mod}_{\mathfrak{S}}(\varphi) \leqslant p-1$ equipped with a $\mathcal{G}_{K_{\infty}}$-equivariant embedding $T_{\mathfrak{S}}^{*}(\mathfrak{M}) \hookrightarrow V_{\mathrm{st}}^{*}(D)$. (See Definition 2.1 for the definition of $\underline{\operatorname{Mod}}_{\mathfrak{S}}(\varphi)^{\leqslant p-1}$.) Then the image of the embedding is $\mathcal{G}_{K}$-stable if and only if $S \otimes_{\varphi, \mathfrak{S}} \mathfrak{M}$ is a strongly divisible $S$-lattice in $S \otimes_{W(k)} D$ in the sense of [7, Definition 2.2.1]. Furthermore, if $D$ does not admit a non-zero weakly admissible quotient pure of slope $p-1$, then we have $T_{\mathfrak{S}}^{*}(\mathfrak{M})=T_{\mathrm{st}}^{*}\left(S \otimes_{\varphi, \mathfrak{S}} \mathfrak{M}\right)$ as $\mathbb{Z}_{p}$-lattices in $V_{\mathrm{st}}^{*}(D)$. 
This conjecture is proved by Tong Liu [21] when $\operatorname{gr}^{w} D_{K}=0$ for all $w \notin[0, p-2]$, so the only unknown case is when $\operatorname{gr}^{0} D_{K} \neq 0$ and $\operatorname{gr}^{p-1} D_{K} \neq 0$.

We include sections to review Kisin theory (Section 2) and various definitions and constructions involving Breuil modules (Section 3). The main results are stated in Section 4, and proved in Section 5. We allow $p$ to be arbitrary prime in order to illustrate the differences when $p=2$, although our proof is not essentially different from Kisin's $[12,13]$ when $p>2$.

\section{Review: Kisin theory}

Let $\mathfrak{S}:=W(k)[[u]]$ where $u$ is a formal variable. Let $\mathscr{O}_{\mathcal{E}}$ be the $p$-adic completion of $\mathfrak{S}\left[\frac{1}{u}\right]$, and set $\mathcal{E}:=\mathscr{O}_{\mathcal{E}}\left[\frac{1}{p}\right]$. Note that $\mathscr{O}_{\mathcal{E}}$ is a complete discrete valuation ring with uniformiser $p$ and $\mathscr{O}_{\mathcal{E}} /(p) \cong k((u))$. We extend the Witt vectors Frobenius map to $\mathfrak{S}$, $\mathscr{O}_{\mathcal{E}}$, and $\mathcal{E}$ by sending $u$ to $u^{p}$, and denote them by $\varphi$. We denote by $\varphi^{*}(\cdot)$ the scalar extension by $\varphi$. We fix an Eisenstein polynomial $E(u) \in W(k)[u]$ such that $E(\pi)=0$ and $E(0)=p$, and view it as an element of $\mathfrak{S}$. Note that this normalization is different from the usual convention, which takes $E(u)$ to be a monic polynomial - although our discussions does not depend upon the choice of normalization of $E(u)$ in any significant way, our convention seems more natural in some places. (See Section 5.1, for example.)

Definition 2.1. For a non-negative integer $h$, a $\varphi$-module of height $\leqslant h$ is a tuple $\left(\mathfrak{M}, \varphi_{\mathfrak{M}}\right)$, where $\mathfrak{M}$ is a finite free $\mathfrak{S}$-module, and $\varphi_{\mathfrak{M}}: \mathfrak{M} \rightarrow \mathfrak{M}$ is a $\varphi$-semilinear endomorphism such that $E(u)^{h}$ annihilates $\operatorname{coker}\left(1 \otimes \varphi_{\mathfrak{M}}: \varphi^{*} \mathfrak{M} \rightarrow \mathfrak{M}\right)$. Let $\underline{\operatorname{Mod}}_{\mathfrak{S}}(\varphi)^{\leqslant h}$ denote the category of $\varphi$-modules of height $\leqslant h$ with the obvious notion of morphisms. When $h=1$, we set $\mathrm{BT}_{/ \mathfrak{S}}^{\varphi}:=\operatorname{Mod}_{\mathfrak{S}}^{\leqslant 1}(\varphi)$.

2.2. Galois representations. Put $R:=\lim _{x \mapsto x^{p}} \mathscr{O}_{\bar{K}} /(p)$. Note that the sequence $\underline{\pi}:=\left\{\pi^{(n)}\right\}$ is an element of $R$, where $\pi^{(n)} \in K_{\infty}$ is the fixed $p^{n}$ th root of the fixed uniformizer $\pi=\pi^{(0)}$. (See the beginning of Section 1.) It follows from [28, Corollaires 3.2.3, 4.3.4] that Frac $R$ is isomorphic to the $\underline{\pi}$-adic completion of $k((\underline{\pi}))^{\text {alg }}{ }^{3}$ Also $R$ is the valuation ring for the $\underline{\pi}$-adic valuation on Frac $R$ (cf. [28, Section 4.1.1]).

We have a $W(k)$-embedding $\mathfrak{S} \hookrightarrow W(R)$ defined by sending $u$ to $[\underline{\pi}]$, where $[\cdot]$ is the Teichmüller lift. This embedding extends to $\mathscr{O}_{\mathcal{E}} \hookrightarrow W(\operatorname{Frac} R)$. Let $\widehat{\mathscr{O}}_{\mathcal{E}}$ ur be the topological closure of the integral closure of $\mathscr{O}_{\mathcal{E}}$ in $W(\operatorname{Frac} R)$, and $\widehat{\mathfrak{S}}^{\text {ur }}$ the topological closure of the integral closure of $\mathfrak{S}$ in $\widehat{\mathscr{O}}_{\mathcal{E}}^{\text {ur }}$. (We use the $p$-adic topology in both cases.) Clearly, $\widehat{\mathfrak{S}}^{\text {ur }}$ and $\widehat{\mathscr{O}}_{\mathcal{E}^{\text {ur }}}$ are subrings of $W(\operatorname{Frac} R)$ stable under $\varphi$ and $\mathcal{G}_{K_{\infty}}$-action (but not under $\mathcal{G}_{K}$-action), and $\widehat{\mathfrak{S}}^{\text {ur }}$ is contained in $W(R)$.

Now we associate, to $\mathfrak{M} \in \underline{\operatorname{Mod}}_{\mathfrak{S}}(\varphi) \leqslant h$, a $\mathbb{Z}_{p}$-module with continuous $\mathcal{G}_{K_{\infty}}$-action, as follows:

$$
T_{\mathfrak{S}}^{*}(\mathfrak{M}):=\operatorname{Hom}_{\mathfrak{S}, \varphi}\left(\mathfrak{M}, \widehat{\mathfrak{S}}^{\mathrm{ur}}\right)
$$

\footnotetext{
${ }^{3}$ To compare the notation of [28] with ours, $R(\bar{K})=\operatorname{Frac} R$ and $X_{K}\left(K_{\infty}\right)=k((\underline{\pi}))$; to see the second equality, note that $X_{K}\left(K_{\infty}\right)$ is a local field of characteristic $p$ with residue field $k$ [28, Proposition 2.2.4], and one can directly check that $\underline{\pi}$ is the uniformizer of $X_{K}\left(K_{\infty}\right)$ by the definition of the valuation [28, Section 2.2.3.3].
} 
where $\mathcal{G}_{K_{\infty}}$ acts through $\widehat{\mathfrak{S}}^{\text {ur }}$. By Proposition 1.8.3 and Section A 1.2 of [10], it follows that $T_{\mathfrak{S}}^{*}(\mathfrak{M})$ is $\mathbb{Z}_{p}$-free with rank equal to the $\mathfrak{S}$-rank of $\mathfrak{M}$, and $T_{\mathfrak{S}}^{*}$ is exact and commutes with direct sums and $\otimes$-products.

For a topological group $\Gamma$ (e.g., $\left.\mathcal{G}_{K}, \mathcal{G}_{K_{\infty}}\right)$, let $\operatorname{Rep}_{\mathbb{Z}_{p}}^{\text {free }}(\Gamma)$ denote the category of finite free $\mathbb{Z}_{p}$-modules with continuous $\Gamma$-action. Let $\operatorname{Rep}_{\text {cris, } \mathbb{Q}_{p}}^{[0, h]}\left(\mathcal{G}_{K}\right)$ and $\operatorname{Rep}_{\mathrm{st}, \mathbb{Q}_{p}}^{[0, h]}\left(\mathcal{G}_{K}\right)$ respectively denote the categories of crystalline and semi-stable $\mathcal{G}_{K}$-representations $V$

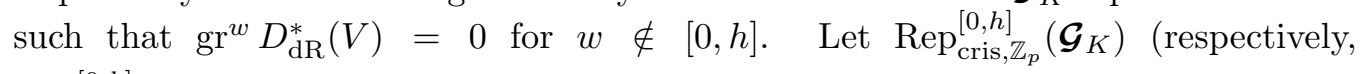
$\left.\operatorname{Rep}_{\mathrm{st}, \mathbb{Z}_{p}}^{[0, h]}\left(\mathcal{G}_{K}\right)\right)$ denote the full subcategory of $\operatorname{Rep}_{\mathbb{Z}_{p}}^{\text {free }}\left(\mathcal{G}_{K}\right)$ consisting of objects $T$ such that $T\left[\frac{1}{p}\right] \in \operatorname{Rep}_{\text {cris, } \mathbb{Q}_{p}}^{[0, h]}\left(\mathcal{G}_{K}\right)$ (respectively, $T\left[\frac{1}{p}\right] \in \operatorname{Rep}_{\mathrm{st}, \mathbb{Q}_{p}}^{[0, h]}\left(\mathcal{G}_{K}\right)$ ). The following non-trivial theorem of Kisin is the starting point of our argument:

\section{Theorem 2.3 (Kisin).}

(1) $\left[12\right.$, Proposition 2.1.12] The contravariant functor $T_{\mathfrak{S}}^{*}: \underline{\operatorname{Mod}}_{\mathfrak{S}}(\varphi) \stackrel{\leqslant h}{\rightarrow} \operatorname{Rep}_{\mathbb{Z}_{p}}^{\text {free }}$ $\left(\mathcal{G}_{K_{\infty}}\right)$ is fully faithful.

(2) There exists a (contravariant) functor $\mathfrak{M}^{*}: \operatorname{Rep}_{\mathrm{st}, \mathbb{Z}_{p}}^{[0, h}\left(\mathcal{G}_{K}\right) \rightarrow \underline{\operatorname{Mod}}_{\mathfrak{S}}(\varphi) \leqslant h$ of $\otimes$-categories such that for any $T \in \operatorname{Rep}_{\mathrm{st}, \mathbb{Z}_{p}}^{[0, h]}\left(\mathcal{G}_{K}\right)$ there exists a natural $\mathcal{G}_{K_{\infty}}$-isomorphism $T \cong T_{\mathfrak{S}}^{*}\left(\mathfrak{M}^{*}(T)\right)$.

(3) $\left[12\right.$, Theorem 2.2.7] Consider the following functor ${ }^{4}$ $\mathfrak{M}^{*} \circ T_{p}:\left\{p\right.$-divisible groups over $\left.\mathscr{O}_{K}\right\} \rightarrow \mathrm{BT}_{/ \mathfrak{S}}^{\varphi} ; G \rightsquigarrow \mathfrak{M}^{*}\left(T_{p}(G)\right)$.

This functor is an anti-equivalence of categories if $p>2$, and induces an anti-equivalence of categories between the corresponding isogeny categories if $p=2$.

Proof. We only explain how to read off (2) from [12]. Kisin constructed the functor $\operatorname{Rep}_{\mathrm{st}, \mathbb{Q}_{p}}^{[0, h]}\left(\mathcal{G}_{K}\right) \rightarrow \underline{\operatorname{Mod}}_{\mathfrak{S}}(\varphi) \leqslant h\left[\frac{1}{p}\right]$ (see [12, Corollary 1.3.15]), and showed that if this functor sends $V \in \operatorname{Rep}_{\mathrm{st}, \mathbb{Q}_{p}}^{[0, h]}\left(\mathcal{G}_{K}\right)$ to the isogeny class containing $\mathfrak{M}^{\prime} \in \underline{\operatorname{Mod}}_{\mathfrak{S}}(\varphi) \leqslant h$ then we have a natural $\mathcal{G}_{K_{\infty}}$-isomorphism $V \cong T_{\mathfrak{S}}^{*}\left(\mathfrak{M}^{\prime}\right)\left[\frac{1}{p}\right]$. (See [12, Proposition 2.1.5].) Finally, the proof of [12, Lemma 2.1.15] shows that for any $\mathcal{G}_{K_{\infty}}$-stable $\mathbb{Z}_{p}$-lattice $T$ in $V \cong T_{\mathfrak{S}}^{*}\left(\mathfrak{M}^{\prime}\right)\left[\frac{1}{p}\right]$ there exists a unique $\varphi$-stable $\mathfrak{S}$-lattice $\mathfrak{M} \subset \mathfrak{M}^{\prime}\left[\frac{1}{p}\right]$ which is of height $\leqslant h$, such that the $\mathbb{Z}_{p}$-lattice $T^{*}(\mathfrak{M})$ in $V$ is precisely $T$. Set $\mathfrak{M}^{*}(T):=\mathfrak{M}$.

Remark 2.4. The functor $\mathfrak{M}^{*}$ is unfortunately not exact although it is exact up to isogeny. ${ }^{5}$ In fact, T. Liu [20, Example 2.5.6] provided an example of a short exact sequence $T^{\bullet}$ of Tate modules of $p$-divisible groups such that $\mathfrak{M}^{*}\left(T^{\bullet}\right)$ is not exact. In that example $T^{\bullet}$ does not extend to a short exact sequence of $p$-divisible groups over $\mathscr{O}_{K},{ }^{6}$ and we will prove later (Section 5.7) that $\mathfrak{M}^{*}$ preserves a short exact sequence of Tate modules whenever it extends to a short exact sequence of $p$-divisible groups.

\footnotetext{
${ }^{4}$ The functor can be defined because $V:=V_{p}(G)$ is a crystalline representation with $\operatorname{gr}^{w} D_{\mathrm{dR}}^{*}(V)=0$ for $w \neq 0,1$.

${ }^{5}$ The author thanks T. Liu for pointing this out to him.

${ }^{6}$ Although by Tate's theorem $[26,(4.2)]$ an exact sequence $0 \rightarrow T_{p}\left(G^{\prime}\right) \rightarrow T_{p}(G) \rightarrow T_{p}\left(G^{\prime \prime}\right) \rightarrow 0$ extends to a sequence $0 \rightarrow G^{\prime} \rightarrow G \rightarrow G^{\prime \prime} \rightarrow 0$ of $p$-divisible groups, this sequence does not have to be exact.
} 
Let us briefly discuss the torsion theory.

Definition 2.5. For a non-negative integer $h$, a torsion $\varphi$-module of height $\leqslant h$ is a tuple $\left(\mathfrak{M}, \varphi_{\mathfrak{M}}\right)$, where $\mathfrak{M}$ is a finitely generated $p^{\infty}$-torsion $\mathfrak{S}$-module with no nonzero $u$-torsion, and $\varphi_{\mathfrak{M}}: \mathfrak{M} \rightarrow \mathfrak{M}$ is a $\varphi$-semilinear endomorphism such that $E(u)^{h}$ annihilates $\operatorname{coker}\left(1 \otimes \varphi_{\mathfrak{M}}: \varphi^{*} \mathfrak{M} \rightarrow \mathfrak{M}\right)$. Let $(\operatorname{Mod} / \mathfrak{S})^{\leqslant h}$ denote the category of torsion $\varphi$-modules of height $\leqslant h$ with the obvious notion of morphisms.

Note that a non-zero $p^{\infty}$-torsion $\mathfrak{S}$-module is of projective dimension $\leqslant 1$ if and only if it has no non-zero $u$-torsion.

Lemma 2.6 ([12, Lemma 2.3.4]). Any $\mathfrak{M} \in(\operatorname{Mod} / \mathfrak{S})^{\leqslant 1}$ fits into a short exact sequence

$$
0 \rightarrow \widetilde{\mathfrak{M}^{\prime}} \stackrel{f}{\rightarrow} \widetilde{\mathfrak{M}} \rightarrow \mathfrak{M} \rightarrow 0,
$$

where $\widetilde{\mathfrak{M}}, \widetilde{\mathfrak{M}^{\prime}} \in \mathrm{BT}_{/ \mathfrak{S}}^{\varphi}$ and all the arrows are $\varphi$-compatible.

To a torsion $\varphi$-module $\mathfrak{M}$ of height $\leqslant h$, we can associate the following torsion $\mathbb{Z}_{p}$-module with continuous $\mathcal{G}_{K_{\infty}}$-action:

$$
T_{\mathfrak{S}}^{*}(\mathfrak{M}):=\operatorname{Hom}_{\mathfrak{S}, \varphi}\left(\mathfrak{M}, \widehat{\mathfrak{S}}^{\mathrm{ur}} \otimes_{\mathbb{Z}_{p}} \mathbb{Q}_{p} / \mathbb{Z}_{p}\right)
$$

One can easily check that the exact sequence (2.6.1) induces the following exact sequence:

$$
0 \rightarrow T_{\mathfrak{S}}^{*}(\widetilde{\mathfrak{M}}) \stackrel{T_{\mathfrak{S}}^{*}(f)}{\longrightarrow} T_{\mathfrak{S}}^{*}\left(\widetilde{\mathfrak{M}^{\prime}}\right) \longrightarrow T_{\mathfrak{S}}^{*}(\mathfrak{M}) \rightarrow 0
$$

where the surjective map is constructed as follows. Identifying $\widetilde{\mathfrak{M}^{\prime}}\left[\frac{1}{p}\right]$ with $\widetilde{\mathfrak{M}}\left[\frac{1}{p}\right]$ by $f\left[\frac{1}{p}\right]$, a $\mathfrak{S}$-linear map $l: \widetilde{\mathfrak{M}}^{\prime} \rightarrow \widehat{\mathfrak{S}}^{\text {ur }}$ can be viewed as $l: \widetilde{\mathfrak{M}} \rightarrow \widehat{\mathfrak{S}}^{\text {ur }} \otimes_{\mathbb{Z}_{p}} \mathbb{Q}_{p}$ and clearly $l \bmod \widehat{\mathfrak{S}}^{\text {ur }}$ factors through $\mathfrak{M} \cong \widetilde{\mathfrak{M}} / \widetilde{\mathfrak{M}}^{\prime}$. The arrow is defined by sending $l$ to $l \bmod \widehat{\mathfrak{S}}^{\mathrm{ur}}$.

\section{Review: Breuil modules}

We recall basic definitions and constructions to work with various $S$-modules introduced by Breuil. All results in this section are "standard" to experts, so we often omit the proof by giving references.

3.1. The ring $S$. Let $S$ be the $p$-adic completion of the divided power envelop of $W(k)[u]$ with respect to the ideal generated by $E(u)$. It can be shown that $S$ can be viewed as a subring of $K_{0}[[u]]$ whose elements are precisely those of the form $\sum_{i \geq 0} a_{i} \frac{u^{i}}{q(i) !}$, where $q(i):=\left\lfloor\frac{i}{e}\right\rfloor$ with $e:=\operatorname{deg} E(u)$, and $a_{i} \in W(k)$ converge to 0 as $i \rightarrow \infty .^{7}$ (In particular, $S$ is naturally a $\mathfrak{S}$-algebra.) We define a differential operator $N:=-u \frac{d}{d u}$ on $S$, and a ring endomorphism $\varphi: S \rightarrow S$ which extends the Witt vector Frobenius map by $\varphi(u)=u^{p}$. We let Fil ${ }^{1} S \subset S$ denote the ideal topologically generated by $E(u)^{i} / i$ ! for $i \geqslant 1$. Since $p \mid \varphi(f)$ for any $f \in \mathrm{Fil}^{1} S$, we can define a $\varphi$-semilinear map $\varphi_{1}:=\frac{\varphi}{p}:$ Fil $^{1} S \rightarrow S$.

\footnotetext{
${ }^{7}$ The proof is by rearranging the terms of $\sum_{i \geqslant 0} b_{i} \frac{E(u)^{i}}{i !}$ and show that the coefficient $a_{i}$ of $\frac{u^{i}}{q(i) !}$ converges, for which boundedness of $\left\{\frac{p^{n}}{n !}\right\}$ suffices. (From $\lim _{i \rightarrow \infty} b_{i}=0$, we deduce $\lim _{i \rightarrow \infty} a_{i}=0$.) In particular, this argument works when $p=2$.
} 
3.2. Strongly divisible $S$-modules. Let $D$ be an admissible filtered $\varphi$-module over $K$ such that $\operatorname{gr}^{w} D_{K}=0$ for $w \neq 0,1$. Let us consider $\mathcal{D}:=S \otimes_{W(k)} D$ equipped with the following structure:

(1) a $\varphi$-linear map $\varphi_{\mathcal{D}}:=\varphi_{S} \otimes \varphi_{D}: S \otimes_{W(k)} D \rightarrow S \otimes_{W(k)} D$;

(2) a derivation $N_{\mathcal{D}}:=N \otimes \operatorname{id}_{D}: S \otimes_{W(k)} D \rightarrow S \otimes_{W(k)} D$ over $N: S \rightarrow S$;

(3) an $S\left[\frac{1}{p}\right]$-submodule Fil ${ }^{1} \mathcal{D}:=\left\{m \in S \otimes_{W(k)} D \mid m \bmod E(u) \in \mathrm{Fil}^{1} D_{K}\right\}$.

See [4] or [7, Section 2.2] for more details (allowing higher Hodge-Tate weights). We now define strongly divisible $S$-lattices in $\mathcal{D}$, following [7, Definition 2.2.1] except that we do not impose any condition ${ }^{8}$ on $D$ when $p=2$ :

Definition 3.2.1. A strongly divisible $S$-lattice in $\mathcal{D}$ is an $S$-submodule $\mathcal{M}$ which satisfies the following properties:

(1) $\mathcal{M}$ is a finite free $S$-module with $\mathcal{M}\left[\frac{1}{p}\right]=\mathcal{D}$;

(2) $\mathcal{M}$ is stable under $\varphi_{\mathcal{D}}$ and $N$;

(3) we have $\varphi_{\mathcal{D}}\left(\mathrm{Fil}^{1} \mathcal{M}\right) \subset p \mathcal{M}$ where $\mathrm{Fil}^{1} \mathcal{M}:=\mathcal{M} \cap \mathrm{Fil}^{1} \mathcal{D}$.

We put $\varphi_{1}:=\frac{1}{p}\left(\left.\varphi_{\mathcal{D}}\right|_{\mathrm{Fil}^{1}} \mathcal{M}\right): \mathrm{Fil}^{1} \mathcal{M} \rightarrow \mathcal{M}$, one can recover $\left.\varphi_{\mathcal{D}}\right|_{\mathcal{M}}$ from $\varphi_{1}$ as follows:

$$
\varphi_{\mathcal{D}}(m)=c_{1}^{-1} \varphi_{1}(E(u) m)
$$

for any $m \in \mathcal{M}$, where $c_{1}:=\varphi(E(u)) / p$, which can be easily seen to be a unit. Note also that $N_{\mathcal{M}}:=\left.N_{\mathcal{D}}\right|_{\mathcal{M}}$ and $\varphi_{1}$ satisfy the following relation called "Griffiths transversality":

$$
N_{\mathcal{M}} \circ \varphi_{1}=c_{1}^{-1} \varphi_{1} \circ\left(E(u) \cdot N_{\mathcal{M}}\right): \mathrm{Fil}^{1} \mathcal{M} \rightarrow \mathcal{M}
$$

We can axiomatize these structures and define strongly divisible S-modules of weight $\leqslant 1$ without mentioning the ambient module $\mathcal{D}$. See [7, Section 2.2] for more details.

3.3. Construction of strongly divisible $\boldsymbol{S}$-modules. Let $\mathfrak{M}$ be an object of either $\mathrm{BT}_{/ \mathfrak{S}}^{\varphi}$ or $(\operatorname{Mod} / \mathfrak{S})^{\leqslant 1}$, and we will define some additional structure on the $S$-module $\mathcal{M}:=S \otimes_{\varphi, \mathfrak{S}} \mathfrak{M}$, which makes $\mathcal{M}$ a strongly divisible $S$-module if $\mathfrak{M}$ is S-free. Using the $S$-linear map $1 \otimes \varphi_{\mathfrak{M}}: \mathcal{M} \cong S \otimes_{\mathfrak{S}}\left(\varphi^{*} \mathfrak{M}\right) \rightarrow S \otimes_{\mathfrak{S}} \mathfrak{M}$, we define an $S$-submodule $\mathrm{Fil}^{1} \mathcal{M} \subset \mathcal{M}$ and $\varphi_{1}: \mathrm{Fil}^{1} \mathcal{M} \rightarrow \mathcal{M}$ as follows:

$$
\begin{gathered}
\mathrm{Fil}^{1} \mathcal{M}:=\left\{x \in \mathcal{M} \mid 1 \otimes \varphi_{\mathfrak{M}}(x) \in \mathrm{Fil}^{1} S \otimes_{\mathfrak{S}} \mathfrak{M} \subset S \otimes_{\mathfrak{S}} \mathfrak{M}\right\}, \\
\varphi_{1}: \mathrm{Fil}^{1} \mathcal{M} \stackrel{1 \otimes \varphi_{\mathfrak{M}}}{\longrightarrow} \mathrm{Fil}^{1} S \otimes_{\mathfrak{S}} \mathfrak{M} \stackrel{\varphi_{1} \otimes 1}{\longrightarrow} S \otimes_{\varphi, \mathfrak{S}} \mathfrak{M}=\mathcal{M} .
\end{gathered}
$$

Let $\varphi_{\mathcal{M}}:=\varphi \otimes \varphi_{\mathfrak{M}}$, and note that $\varphi_{1}=\frac{1}{p}\left(\left.\varphi_{\mathcal{M}}\right|_{\mathrm{Fil}^{1} \mathcal{M}}\right)$.

Example 3.3.2. Consider $\mathfrak{M}=\mathfrak{S}$ equipped with $\varphi_{\mathfrak{M}}=E(u) \cdot \varphi$. Then $\mathcal{M}=$ Fil $^{1}$ $\mathcal{M}=S$ and $\varphi_{1}(1)=\varphi(E(u)) / p$.

This functor $S \otimes_{\varphi, \mathfrak{S}}(\cdot)$ is obviously faithful. We now claim that this functor is also exact.

\footnotetext{
${ }^{8}$ I.e., we do not assume that $D$ has no non-zero weakly admissible quotient pure of slope 1 as in [7, Section 2.1].
} 
Lemma 3.3.3. Assume that we have a $\varphi$-compatible short exact sequence $\left(\mathfrak{M}^{\bullet}\right)$ : $0 \rightarrow \mathfrak{M}^{\prime} \rightarrow \mathfrak{M} \rightarrow \mathfrak{M}^{\prime \prime} \rightarrow 0$ where $\mathfrak{M}^{\prime}, \mathfrak{M}$, and $\mathfrak{M}^{\prime \prime}$ are objects of either $(\mathrm{Mod} / \mathfrak{S})^{\leqslant 1}$ or $\mathrm{BT}_{/ \mathfrak{S}}^{\varphi}$. Then the sequence $S \otimes_{\varphi, \mathfrak{S}}\left(\mathfrak{M}^{\bullet}\right)$ is short exact, respects $\varphi_{1}$ 's, and induces a short exact sequence on $\mathrm{Fil}^{1}$ 's.

Proof. The only case when the exactness of $S \otimes_{\varphi, \mathfrak{S}}\left(\mathfrak{M}^{\bullet}\right)$ is possibly non-trivial is when $\mathfrak{M}^{\prime \prime} \in(\operatorname{Mod} / \mathfrak{S})^{\leqslant 1}$. In this case, it is enough to show $\operatorname{Tor}_{1}^{\mathfrak{S}}\left(\mathfrak{M}^{\prime \prime}, S\right)=0$. But since $\mathfrak{M}^{\prime \prime}$ is a successive extension of $\mathfrak{S} / p \mathfrak{S}$ as a $\mathfrak{S}$-module, it is enough to show $\operatorname{Tor}_{1}^{\mathfrak{S}}(\mathfrak{S} / p \mathfrak{S}, S)=0$, which is obvious. The rest of the claim can be directly checked from the definition ( $c f$. equations (3.3.1a) and (3.3.1b)).

Suppose $\mathfrak{M} \in \mathrm{BT}_{/ \mathfrak{S}}^{\varphi}$. Set $D:=D_{\text {cris }}^{*}\left(V_{p}\left(G^{\prime}\right)\right)$ for a $p$-divisible group $G^{\prime}$ over $\mathscr{O}_{K}$ which corresponds to $\mathfrak{M}$ up to isogeny by Theorem $2.3(3)$. We write $\mathcal{D}:=S \otimes_{W(k)} D$ and $\mathcal{M}:=S \otimes_{\varphi, \mathfrak{S}} \mathfrak{M}$.

Proposition 3.3.4. We use the notation as above. Then there exists a natural $S$ linear embedding $\eta: \mathcal{M} \hookrightarrow \mathcal{D}$ such that $\varphi_{\mathcal{D}} \circ \eta=\eta \circ \varphi_{\mathcal{M}}, N_{\mathcal{D}}(\eta(\mathcal{M})) \subset \eta(\mathcal{M})$ and $\eta\left(\mathrm{Fil}^{1} \mathcal{M}\right)=\eta(\mathcal{M}) \cap \mathrm{Fil}^{1} \mathcal{D}$.

Proof. The assertion except $N_{\mathcal{D}}(\mathcal{M}) \subset \mathcal{M}$ follows from [21, Corollary 3.2.3] using the rigid analytic construction [12, Corollary 1.3.15] relating $D$ and $\mathfrak{M}$. Now one can apply [6, Proposition 5.1.3 $]^{9}$ to conclude.

Corollary 3.3.5. For any $\mathfrak{M} \in(\operatorname{Mod} / \mathfrak{S})^{\leqslant 1}$, there exists a unique differential operator $N_{\mathcal{M}}: \mathcal{M} \rightarrow \mathcal{M}$ over $N: S \rightarrow S$ such that the "Griffiths transversality" (i.e., equation (3.2.2)) holds and $N_{\mathcal{M}} \equiv 0 \bmod I_{0} \mathcal{M}$ where $I_{0} \subset S$ is the ideal topologically generated by $\frac{u^{e i}}{i !}$ for $i \geqslant 0$.

Proof. By Lemma 2.6, we can find $\widetilde{\mathfrak{M}}, \widetilde{\mathfrak{M}^{\prime}} \in \mathrm{BT}_{/ \mathfrak{S}}^{\varphi}$ with $\mathfrak{M} \cong \widetilde{\mathfrak{M}} / \widetilde{\mathfrak{M}^{\prime}}$. By Proposition 3.3.4, both $\widetilde{\mathcal{M}}:=S \otimes_{\varphi, \mathfrak{S}} \widetilde{\mathfrak{M}}$ and $\widetilde{\mathcal{M}}{ }^{\prime}:=S \otimes_{\varphi, \mathfrak{S}^{\prime}} \widetilde{\mathfrak{M}^{\prime}}$ are strongly divisible $S$-lattices in $S \otimes_{W(k)} D$ for some filtered $\varphi$-module $D$. Therefore, $N_{\mathcal{M}}:=N_{\widetilde{\mathcal{M}}} \bmod \widetilde{\mathcal{M}}^{\prime}$ is well defined and satisfies all the desired properties. The uniqueness follows from the proof of [7, Lemma 3.2.1].

3.4. Crystalline period ring. Let us recall the construction of $A_{\text {cris }}$. For more complete discussions, see [11, Sections 1, 2].

There exists a "canonical lift" $\theta: W(R) \rightarrow \mathscr{O}_{\bar{K}}$ of the first projection $R \rightarrow \mathscr{O}_{\bar{K}} /(p)$, which is $\mathcal{G}_{K}$-equivariant for the natural actions on both sides. We define $A_{\text {cris }}$ as the $p$-adic completion of the divided power envelop of $W(R)$ with respect to $\operatorname{ker}(\theta)$. The Witt vector Frobenius map and the $\mathcal{G}_{K}$-action on $W(R)$ extend to $A_{\text {cris }}$. We let $\mathrm{Fil}^{1} A_{\text {cris }}$ be the ideal topologically generated by $\frac{1}{i !}(\operatorname{ker} \theta)^{i}$ for $i \geqslant 1$ (under the $p$-adic topology $)$. We have $\varphi\left(\right.$ Fil $\left.^{1} A_{\text {cris }}\right) A_{\text {cris }} \subset p A_{\text {cris }}$, so we can define $\varphi_{1}:=\frac{\varphi}{p}:$ Fil $^{1} A_{\text {cris }} \rightarrow$ $A_{\text {cris. }}$.

The embedding $\mathfrak{S} \hookrightarrow W(R)$ constructed in Section 2.2 extends to $S \hookrightarrow A_{\text {cris }}$ by the universal property of divided powers envelop. This map is $\varphi$-compatible and $\mathcal{G}_{K_{\infty}}$-invariant (but not $\mathcal{G}_{K}$-invariant).

\footnotetext{
${ }^{9}$ The proof of [6, Proposition 5.1.3] works even when $p=2$.
} 
Let us consider the sequence $\underline{\epsilon}:=\left\{\epsilon^{(n)}\right\}_{n \geqslant 0}$ of cocycle $\epsilon^{(n)}: \mathcal{G}_{K} \rightarrow R^{\times}$defined as follows:

$$
\epsilon^{(n)}(g):=g \cdot \pi^{(n)} / \pi^{(n)}
$$

for any $g \in \mathcal{G}_{K}$. Clearly $\underline{\epsilon}(g)$ defines an element in $R$, and the $n$th component $\epsilon^{(n)}(g)$ is a (not necessarily primitive) $p^{n}$ th root of unity. One can easily check that the following formula defines an element in $\mathrm{Fil}^{1} A_{\text {cris }}$ :

$$
t_{g}:=\log [\underline{\epsilon}(g)]=\sum_{n=1}^{\infty} \frac{(-1)^{n-1}([\underline{\epsilon}(g)]-1)^{n}}{n},
$$

where $g \in \mathcal{G}_{K}$ and [.] denotes the Teichmüller lift.

Remark 3.4.3. When $p=2$, one can show that $t_{g} / 2 \in \mathrm{Fil}^{1} A_{\text {cris }}$ (by modifying the argument in $\left[11\right.$, Section 5.2.9]), so we have $\gamma_{n}\left(t_{g} / 2\right) \in$ Fil $^{1} A_{\text {cris }}$ since Fil ${ }^{1} A_{\text {cris }}$ has a divided power structure. It follows that for any $p$ the sequence $\gamma_{n}\left(t_{g}\right)$ converges to 0 as for the $p$-adic topology in $A_{\text {cris }}$. In particular, we have an equality $[\underline{\epsilon}(g)]=$ $\sum_{n=0}^{\infty} \gamma_{n}\left(t_{g}\right)\left(=\exp \left(t_{g}\right)\right)$ in $A_{\text {cris }}$.

Choose $g \in \mathcal{G}_{K}$ such that $g \pi^{(1)} \neq \pi^{(1)}$. Set $[\underline{\epsilon}]:=[\underline{\epsilon}(g)]$ and $t:=t_{g}$ as in (3.4.2). For any integer $n \geqslant 0$ we set $t^{\{n\}}:=t^{n} /\left(p^{q(n)} q(n) !\right)$ where $q(n):=\lfloor n /(p-1)\rfloor$. Note that $t^{\{n\}} \in A_{\text {cris }}$, and if $p=2$ then $t^{\{n\}}=\gamma_{n}(t / 2)$.

Consider the following subring $\mathfrak{a}_{\text {cris }}$ of Frac $W(R)[[T]]$, where $T$ is a formal variable:

$$
\mathfrak{a}_{\text {cris }}:=\left\{\sum_{n=0}^{\infty} a_{n} T^{\{n\}} \mid a_{n} \in W(R) \text { such that } p \text {-adically } a_{n} \rightarrow 0 .\right\} .
$$

Now, one can give the following explicit description of $A_{\text {cris }}$. See [11, Sections 5.2.75.2.9] for the proof.

Theorem 3.4.4. The $W(R)$-algebra map $\mathfrak{a}_{\text {cris }} \rightarrow A_{\text {cris }}$ defined by $T^{\{n\}} \mapsto t^{\{n\}}$ is surjective and the kernel is generated by $[\underline{\epsilon}]-\sum_{n=0}^{\infty} \gamma_{n}(T)$.

3.5. Construction of Galois-stable $\mathbb{Z}_{\boldsymbol{p}}$-lattices. Let $\mathfrak{M}$ be an object of either $\mathrm{BT}_{/ \mathfrak{S}}^{\varphi}$ or $(\operatorname{Mod} / \mathfrak{S})^{\leqslant 1}$, and put $\mathcal{M}:=S \otimes_{\varphi, \mathfrak{S}} \mathfrak{M}$ with additional structure defined in Section 3.3. Consider the following $\mathbb{Z}_{p}$-module to which we will endow a natural continuous $\mathcal{G}_{K}$-action:

$$
\begin{array}{r}
T_{\mathrm{st}}^{*}(\mathcal{M}):=\operatorname{Hom}_{S, \varphi_{1}, \mathrm{Fil}^{1}}\left(\mathcal{M}, A_{\mathrm{cris}}\right), \text { if } \mathfrak{M} \in \mathrm{BT}_{/ \mathfrak{S}}^{\varphi}, \\
T_{\mathrm{st}}^{*}(\mathcal{M}):=\operatorname{Hom}_{S, \varphi_{1}, \mathrm{Fil}^{1}}\left(\mathcal{M}, A_{\text {cris }} \otimes_{\mathbb{Z}_{p}} \mathbb{Q}_{p} / \mathbb{Z}_{p}\right), \text { if } \mathfrak{M} \in(\operatorname{Mod} / \mathfrak{S})^{\leqslant 1} .
\end{array}
$$

We let $\mathcal{G}_{K_{\infty}}$ act through the second argument $A_{\text {cris }}$. The $\mathcal{G}_{K}$-action is more subtle to describe because the embedding $S \rightarrow A_{\text {cris }}$ is only $\mathcal{G}_{K_{\infty}}$-stable, not $\mathcal{G}_{K}$-stable. Let us first define a $\mathcal{G}_{K}$-action on $A_{\text {cris }} \otimes_{S} \mathcal{M}$ using the differential operator $N_{\mathcal{M}}$ as follows.

$$
g \cdot(a \otimes x):=g(a) \sum_{i=0}^{\infty} \gamma_{i}\left(t_{g}\right) \otimes N_{\mathcal{M}}^{i}(x),
$$

for $g \in \mathcal{G}_{K}, a \in A_{\text {cris }}$, and $x \in \mathcal{M}$. Here, $t_{g} \in \mathrm{Fil}^{1} A_{\text {cris }}$ is as in (3.4.2), and $\gamma_{i}\left(t_{g}\right)$ is the standard $i$ th divided power; i.e., $\gamma_{i}\left(t_{g}\right):=t_{g}^{i} / i$ ! if $i>0$ and $\gamma_{0}\left(t_{g}\right):=1$ (even when $\left.t_{g}=0\right)$. By [21, Lemma 5.1.1], the sum (3.5.2a) converges and gives a $\mathcal{G}_{K}$-action, 
which respects $\varphi$ and the natural filtration on $A_{\text {cris }} \otimes_{S} \mathcal{M}$. Note also that we recover the natural $\mathcal{G}_{K_{\infty}}$-action on $A_{\text {cris }} \otimes_{S} \mathcal{M}$ since $t_{g}=0$ for $g \in \mathcal{G}_{K_{\infty}}$.

For any $f \in T_{\mathrm{st}}^{*}(\mathcal{M})$, we $A_{\text {cris }}$-linearly extend $f$ to $A_{\text {cris }} \otimes_{S} \mathcal{M} \rightarrow A_{\text {cris }}$. Now it is clear that the following formula defines a continuous action of $g \in \mathcal{G}_{K}$ on $T_{\mathrm{st}}^{*}(\mathcal{M})$ :

$$
g \cdot f: x \mapsto g \cdot\left(f\left(g^{-1}(1 \otimes x)\right)\right), \text { for } x \in \mathcal{M} .
$$

The following lemma is straightforward from [21, Lemma 5.2.1 $]^{10}$ and [7, Proposition 2.2.5], using that $T_{\mathrm{st}}^{*}(\mathcal{M})$ is $p$-adically separated and complete.

Lemma 3.5.3. Let $D$ be as in Section 3.1. If $\mathcal{M}$ is a strongly divisible $S$-lattice in $S \otimes_{W(k)} D$ then there exists a natural $\mathcal{G}_{K}$-equivariant injective map $T_{\mathrm{st}}^{*}(\mathcal{M}) \hookrightarrow$ $V_{\text {cris }}^{*}(D)$, whose image is a full $\mathbb{Z}_{p}$-lattice.

The following lemma on the exactness of $T_{\mathrm{st}}^{*}$ will be needed in Section 5 .

Lemma 3.6. Let $D_{i}$ for $i=1,2,3$ be admissible filtered $\varphi$-modules over $K$ with $\operatorname{gr}^{w}\left(D_{i}\right)_{K}=0$ for $w \neq 0,1$, and let $\mathcal{M}_{i}$ be a strongly divisible $S$-lattices in $S \otimes_{W(k)} D_{i}$. For any short exact sequence $0 \rightarrow \mathcal{M}_{1} \rightarrow \mathcal{M}_{2} \rightarrow \mathcal{M}_{3} \rightarrow 0$, the following sequence is short exact:

$$
0 \rightarrow T_{\mathrm{st}}^{*}\left(\mathcal{M}_{3}\right) \rightarrow T_{\mathrm{st}}^{*}\left(\mathcal{M}_{2}\right) \rightarrow T_{\mathrm{st}}^{*}\left(\mathcal{M}_{1}\right) \rightarrow 0 .
$$

Proof. The left exactness of sequence (3.6.1) is clear from the definition of $T_{\mathrm{st}}^{*}$, and the right exactness can be check after reducing it modulo $p$. Moreover, it suffices to show the left exactness of the mod $p$ reduction of sequence (3.6.1), as the $\mathbb{F}_{p}$-dimension of each term adds up correctly.

Now, the natural map $T_{\text {st }}^{*}\left(\mathcal{M}_{i}\right) /(p) \rightarrow T_{\text {st }}^{*}\left(\mathcal{M}_{i} /(p)\right)$ is injective, and the natural map $T_{\mathrm{st}}^{*}\left(\mathcal{M}_{3} /(p)\right) \rightarrow T_{\mathrm{st}}^{*}\left(\mathcal{M}_{2} /(p)\right)$ induced by $\mathcal{M}_{2} /(p) \rightarrow \mathcal{M}_{3} /(p)$ is injective as $T_{\mathrm{st}}^{*}$ is left exact in the torsion case as well. This shows the left exactness the mod $p$ reduction of sequence (3.6.1), hence the lemma follows.

\subsection{Relation with Galois representations coming from Kisin modules.} Note that the natural map $W(R) \rightarrow A_{\text {cris }}$ is injective [11, Section 2.3.3], so we may view $\widehat{\mathfrak{S}}^{\text {ur }}$ as a subring of $A_{\text {cris }}$ (cf. Section 2.2 ). Now consider the following natural $\mathcal{G}_{K_{\infty}}$-equivariant morphism:

$$
\imath: T_{\mathfrak{S}}^{*}(\mathfrak{M})=\operatorname{Hom}_{\mathfrak{S}, \varphi}\left(\mathfrak{M}, \widehat{\mathfrak{S}}^{\mathrm{ur}}\right) \rightarrow \operatorname{Hom}_{S, \varphi_{1}, \mathrm{Fil}^{1}}\left(\mathcal{M}, A_{\text {cris }}\right)=T_{\mathrm{st}}^{*}(\mathcal{M})
$$

where the arrow in the middle is defined as follows: the arrow in (3.7.1) takes $f$ : $\mathfrak{M} \rightarrow \widehat{\mathfrak{S}}^{\text {ur }}$ to $\tilde{f}: \mathcal{M}=S \otimes_{\varphi, \mathfrak{S}} \mathfrak{M} \rightarrow A_{\text {cris }}$, which is obtained by $S$-linearly extending $\mathfrak{M} \stackrel{f}{\rightarrow} \widehat{\mathfrak{S}}^{\text {ur }} \stackrel{\varphi}{\rightarrow} A_{\text {cris }}$, where we view $S$ as a $\mathfrak{S}$-algebra via $\varphi: \mathfrak{S} \rightarrow S$. One can check that if $f$ respects $\varphi$, then $\tilde{f}$ respects $\varphi_{1}$ and takes $\mathrm{Fil}^{1} \mathcal{M}$ to $\mathrm{Fil}^{1} A_{\text {cris. }}$. This map (3.7.1) is clearly $\mathcal{G}_{K_{\infty}}$-equivariant, and one can direct check that the map (3.7.1) respects the natural embeddings of the source and the target into $V .^{11}$

Unfortunately, when $p=2$ the map $\imath$ defined in equation (3.7.1) is not in general an isomorphism. ${ }^{12}$ We prove, instead, that the image of $\imath$ is a $\mathcal{G}_{K}$-stable submodule

\footnotetext{
${ }^{10}$ The assumption that $p>2$ under which [21] was written is not relevant to this result.

${ }^{11}$ See the proof of $\left[12\right.$, Proposition 2.1.5] for $T_{\mathfrak{S}}^{*}(\mathfrak{M}) \hookrightarrow V_{\text {cris }}^{*}(D)$, and the proofs of [7, Proposition 2.2.5] and [21, Lemma 5.2.1] for $T_{\mathrm{st}}^{*}(\mathcal{M}) \hookrightarrow V_{\text {cris }}^{*}(D)$.

${ }^{12}$ If $p>2$ then map $\imath$ is an isomorphism. The proof can be found in the proof of [12, Theorem 2.2.7], and it can also be deduced from Proposition 5.4 below.
} 
in $T_{\mathrm{st}}^{*}(\mathcal{M})$, which suffices for our purpose (cf. Proposition 4.5). This claim will proved in Section 5.

\section{Statement of the main result}

Let $G$ be a $p$-divisible group over $\mathscr{O}_{K}$, and $T:=T_{p}(G)$ its Tate module. Recall that we have the following functor defined in Theorem 2.3(3):

$$
\left\{p \text {-divisible groups over } \mathscr{O}_{K}\right\} \rightarrow \mathrm{BT}_{/ \mathfrak{S}}^{\varphi} ; G \rightsquigarrow \mathfrak{M}_{G}:=\mathfrak{M}^{*}\left(T_{p}(G)\right) \text {. }
$$

By the theorems of Tate $[26,(4.2)]$ and Kisin (Theorem 2.3(2)), the functor $\mathfrak{M}^{*} \circ T_{p}$ is fully faithful.

Theorem 4.1. Assume that $\mathscr{O}_{K}$ is a 2-adic discrete valuation ring with perfect residue field. Then the functor $\mathfrak{M}^{*} \circ T_{p}$ is an anti-equivalence of categories. Furthermore, for any $p$-divisible group $G$ over $\mathscr{O}_{K}$ and $\mathfrak{M}_{G}:=\mathfrak{M}^{*}\left(T_{p}(G)\right)$, there is a natural $\mathcal{G}_{K_{\infty}}$ isomorphism $T_{p}(G) \cong T_{\mathfrak{S}}^{*}\left(\mathfrak{M}_{G}\right)$.

Note that the second assertion is automatic from the property of $\mathfrak{M}^{*}$ stated in Theorem 2.3(2).

Now, let us state the relation of this classification with crystalline Dieudonné theory. Let $G$ be a $p$-divisible group over $\mathscr{O}_{K}$ with residue characteristic $p=2$, $\mathfrak{M}_{G}:=\mathfrak{M}^{*}\left(T_{p}(G)\right)$, and $D:=D_{\text {cris }}^{*}\left(V_{p}(G)\right)$. Let $\mathbb{D}^{*}(G)$ denote the contravariant Dieudonné crystal. (See, for example, [24] for the definition.) Then, $\mathbb{D}^{*}(G)(S)$ and $S \otimes_{\varphi, \mathfrak{S}} \mathfrak{M}_{G}$ are strongly divisible $S$-lattices in $S \otimes_{W(k)} D$. (See Section 3.3 and [12, Section A] for more details.) We will prove the following proposition in Sections 5.6 and 5.7 .

Proposition 4.2. We use the same notation as above.

(1) We have $\mathbb{D}^{*}(G)(S)=S \otimes_{\varphi, \mathfrak{S}} \mathfrak{M}_{G}$ as strongly divisible $S$-lattices in $S \otimes_{W(k)} D$.

(2) The functor $\mathfrak{M}^{*} \circ T_{p}$ takes a short exact sequence of $p$-divisible groups to a short exact sequence in $\mathrm{BT}_{/ \mathfrak{S}}^{\varphi}$.

The proof of (2) uses (1) and the exactness of $\mathbb{D}^{*}$; as explained in Remark 2.4 we cannot hope to prove the exactness of $\mathfrak{M}^{*} \circ T_{p}$ by a purely linear algebraic method. We also prove a stronger exactness assertion which is useful in practice; namely, that any quasi-inverse of $\mathfrak{M}^{*} \circ T_{p}$ should also be an exact functor.

Raynaud's theorem [1, Théorème 3.1.1] implies that for any $p$-power order finite flat group scheme $H$ over $\mathscr{O}_{K}$, there exists an isogeny $f: G \rightarrow G^{\prime}$ of $p$-divisible groups over $\mathscr{O}_{K}$ such that $H \cong \operatorname{ker}(f)$. We set $\mathfrak{M}_{H}:=\operatorname{coker}\left(\left(\mathfrak{M}^{*} \circ T_{p}\right)(f)\right)$, and one can check that $\mathfrak{M}_{H} \in(\operatorname{Mod} / \mathfrak{S})^{\leqslant 1}$ does not depend on the choice of $f$ up to isomorphism, using Proposition 4.2(2). One can also define the (contravariant) Dieudonné crystal $\mathbb{D}^{*}(H)$ in such a way that we have the following natural short exact sequence:

$$
0 \rightarrow \mathbb{D}^{*}\left(G^{\prime}\right)(S) \stackrel{\mathbb{D}^{*}(f)}{\longrightarrow} \mathbb{D}^{*}(G)(S) \rightarrow \mathbb{D}^{*}(H)(S) \rightarrow 0
$$

(See Définition 3.1.5 and Théorème 3.1.2 of [1] for more details.) From this exact sequence one can define Fil $^{1}$ and $\varphi_{1}$ on $\mathbb{D}^{*}(H)(S),{ }^{13}$ which do not depend on the choice of $f$.

\footnotetext{
${ }^{13}$ I.e., $\mathbb{D}^{*}(H)(S) \in(\operatorname{Mod} / S)$ in the sense of [6, Section 2.1.1], but allowing $p=2$.
} 
Corollary 4.3. There exists an exact anti-equivalence of categories $H \rightsquigarrow \mathfrak{M}_{H}$ from the category of p-power order finite flat group schemes over $\mathscr{O}_{K}$ to $(\operatorname{Mod} / \mathfrak{S})^{\leqslant 1}$ which satisfies the following properties:

(1) There is a natural $\mathcal{G}_{K_{\infty}}$-isomorphism $H(\bar{K}) \cong T_{\mathfrak{S}}^{*}\left(\mathfrak{M}_{H}\right)$, where $T_{\mathfrak{S}}^{*}$ is defined in equation (2.6.2).

(2) For any $p$-divisible group $G$ over $\mathscr{O}_{K}$, we have a natural isomorphism $\mathfrak{M}^{*}$ $\left(T_{p}(G)\right) \cong \lim _{n} \mathfrak{M}_{G\left[p^{n}\right]}$, where the projective limit is taken with respect to the map induced by the natural inclusions $G\left[p^{n}\right] \hookrightarrow G\left[p^{n+1}\right]$.

(3) There exists a natural isomorphism $S \otimes_{\varphi, \mathfrak{S}} \mathfrak{M}_{H} \cong \mathbb{D}^{*}(H)(S)$ which respects $\mathrm{Fil}^{1}$ and $\varphi_{1}$.

Proof. Following the same proof of [12, Theorem 2.3.5] one can deduce the corollary from Theorem 4.1, Proposition 4.2, and Lemma 2.6 (with the discussion following it), possibly except the exactness of the functor $H \rightsquigarrow \mathfrak{M}_{H}$, which is proved in Proposition 5.7.2. Note that the argument crucially uses the exactness of the functor $\mathfrak{M} \circ T_{p}$.

This concludes the proof of Conjecture 1.1. See the introduction (Section 1) for previous results on this conjecture.

It was observed by Breuil [7, Theorem 3.4.3] that the following corollary is a formal consequence $^{14}$ of Corollary 4.3. We refer to loc.cit for the proof.

Corollary 4.4. The functor $\left.T \rightsquigarrow T\right|_{\mathcal{G}_{K_{\infty}}}$ from the category of $p^{\infty}$-torsion $\mathcal{G}_{K^{-}}$ representations coming from some finite flat group scheme over $\mathscr{O}_{K}$ to the category of $p^{\infty}$-torsion $\mathcal{G}_{K_{\infty}}$-representations is fully faithful.

Let us sketch the strategy of the proof of Theorem 4.1, which can be thought of as extending [12, Section 2.2] to the case when $p=2$. The only assertion that needs to be proved is the essential surjectivity of $\mathfrak{M}^{*} \circ T_{p}$; i.e. given any $\mathfrak{M} \in \mathrm{BT}_{/ \mathfrak{S}}^{\varphi}$, we need to produce a $p$-divisible group $G$ over $\mathscr{O}_{K}$ such that $\mathfrak{M} \cong \mathfrak{M}^{*}\left(T_{p}(G)\right)$. For any given $\mathfrak{M} \in \mathrm{BT}_{/ \mathfrak{S}}^{\varphi}$, Kisin's theorem (Theorem 2.3(3)) provides a $p$-divisible group $G^{\prime}$ over $\mathscr{O}_{K}$ such that $\mathfrak{M}\left[\frac{1}{p}\right] \cong \mathfrak{M}^{*}\left(T_{p}\left(G^{\prime}\right)\right)\left[\frac{1}{p}\right]$. Set $V:=T_{p}\left(G^{\prime}\right)\left[\frac{1}{p}\right]$ and note that $T_{\mathfrak{S}}^{*}(\mathfrak{M})$ embeds into $V$ as a $\mathcal{G}_{K_{\infty}}$-stable $\mathbb{Z}_{p}$-lattice. The main step is to show the following proposition:

Proposition 4.5. With the notation as above, the $\mathcal{G}_{K_{\infty}}$-stable $\mathbb{Z}_{p}$-lattice $T$ in $V$ is $\mathcal{G}_{K}$-stable.

Granting this proposition, $T$ comes from a $p$-divisible group over $\mathscr{O}_{K}$ by Raynaud's "limit theorem" [25, Proposition 2.3.1], so we obtain Theorem 4.1.

The proof of Proposition 4.5 (which will be given in Section 5) is not straightforward since Breuil's theory of strongly divisible $S$-lattices does not work nicely when $p=2$, but fortunately it does not fail too badly. (See Propositions 5.4 and 5.5 for more details.) Interestingly, we do not use Proposition 4.2 in order to prove Proposition 4.5 or Theorem 4.1.

\footnotetext{
${ }^{14}$ Since we have the classification of any $p$-power order finite flat group schemes, not just the ones killed by $p$, the dévissage step in the proof of [7, Theorem 3.4.3] is unnecessary.
} 
Remark 4.6. Kisin's construction of integral canonical models for Shimura varieties of abelian type [15] has a few technical restrictions when $p=2$, and one of the main reasons is that at the time of [15] we did not have Theorem 4.1 and Proposition 4.2 when $p=2$. (See [15, Theorem 1.4.2, Corollary 1.4.3] for more details.) The 2-adic case causes another complication (of different nature) when the reductive group $G$ has a factor of type B $[15 \text {, Lemma 2.3.1 }]^{15}$, so one may expect that the main result of this paper can be used to construct 2-adic integral models for Shimura varieties of abelian type under some mild restriction on the reductive group $G$. Unfortunately, there is one more deformation argument [15, Proposition 1.5.8] which does not generalize to the 2-adic case. This problem, however, does not seem insurmountable, and it seems that the main result of this paper has removed the main obstacle for generalizing [15] to the 2-adic case.

\section{Proof of the main results}

In this section, we prove Proposition 4.5 (hence, Theorem 4.1) and Proposition 4.2. The result is known when $p>2$ by Kisin [12, Section 2.2] or when $\mathfrak{M}$ is $\varphi$-unipotent (cf. Section 5.1) by Kisin [13, Proposition 1.2.7], and our proof can be viewed as a modification of these arguments. A similar approach to ours (namely, classifying connected and étale $p$-divisible groups separately and handling the general case by connected-étale sequences) is also found in [3] and [8] in their study of Dieudonné crystals, and in [29] where display theory is extended to general $p$-divisible groups.

5.1. We first need to introduce the linear algebra counterparts of étale, connected, multiplicative-type and unipotent finite flat group schemes and $p$-divisible groups over $\mathscr{O}_{K}$, respectively. Let $\mathfrak{M}$ be an object either in $(\operatorname{Mod} / \mathfrak{S})^{\leqslant 1}$ or in $\mathrm{BT}_{/ \mathfrak{S}}^{\varphi}$. Since the linearization $1 \otimes \varphi_{\mathfrak{M}}: \varphi^{*} \mathfrak{M} \rightarrow \mathfrak{M}$ is injective with cokernel killed by $E(u)$, there exists a unique map $\psi_{\mathfrak{M}}: \mathfrak{M} \rightarrow \varphi^{*} \mathfrak{M}$ such that $\psi_{\mathfrak{M}} \circ\left(1 \otimes \varphi_{\mathfrak{M}}\right)$ and $\left(1 \otimes \varphi_{\mathfrak{M}}\right) \circ \psi_{\mathfrak{M}}$ are given by multiplication by $E(u)$.

We first define the analog of Cartier duality $\mathfrak{M}^{\vee}$ as follows. We set the underlying $\mathfrak{S}$-module to be $\mathfrak{M}^{\vee}:=\operatorname{Hom}_{\mathfrak{S}}(\mathfrak{M}, \mathfrak{S})$ if $\mathfrak{M} \in \mathrm{BT}_{/ \mathfrak{S}}^{\varphi}$, and $\mathfrak{M}^{\vee}:=\operatorname{Hom}_{\mathfrak{S}}\left(\mathfrak{M}, \mathfrak{S} \otimes_{\mathbb{Z}_{p}}\right.$ $\left.\mathbb{Q}_{p} / \mathbb{Z}_{p}\right)$ if $\mathfrak{M} \in(\operatorname{Mod} / \mathfrak{S})^{\leqslant 1}$, and we define $\varphi_{\mathfrak{M} \vee}$ so that $1 \otimes \varphi_{\mathfrak{M} \vee}$ is the dual of $\psi_{\mathfrak{M}}$. One can check that $T_{\mathfrak{S}}^{*}\left(\mathfrak{M}^{\vee}\right) \cong\left(T_{\mathfrak{S}}^{*}(\mathfrak{M})\right)^{*} \otimes\left(\chi_{\text {cyc }} \mid \mathcal{G}_{K_{\infty}}\right)$, so for a $p$-divisible group $G$ over $\mathscr{O}_{K}$ we have $\mathfrak{M}^{*}\left(T_{p}(G)\right)^{\vee} \cong \mathfrak{M}^{*}\left(T_{p}\left(G^{\vee}\right)\right)$ where $G^{\vee}$ is the Cartier dual of G. (This essentially follows from $\mathfrak{M}^{*}\left(\mathbb{Z}_{p}(1)\right) \cong(\mathfrak{S}, E(u) \varphi)$; indeed, by [12, Corollary 1.3.15] the isogeny class of $(\mathfrak{S}, E(u) \varphi)$ corresponds to the admissible filtered $\varphi$-module $\left(W(k)\left[\frac{1}{p}\right], E(0) \varphi\right)$, and from our normalization we have $E(0)=p$.) The analogous statement for finite flat group schemes is deduced from this using Proposition $4.2(2)$ which will be proved later.

We say $\mathfrak{M}$ is étale if $1 \otimes \varphi_{\mathfrak{M}}$ is an isomorphism, and of multiplicative type if $\mathfrak{M}^{\vee}$ is étale (i.e., if $\psi_{\mathfrak{M}}$ is an isomorphism). We say $\mathfrak{M}$ is $\varphi$-nilpotent if $\varphi_{\mathfrak{M}}^{n}(\mathfrak{M}) \subset \mathfrak{m}_{\mathfrak{S}} \cdot \mathfrak{M}$ for

\footnotetext{
${ }^{15}$ In concrete cases, this type B restriction is not so serious; this restriction arises when finding a suitable embedding $G \hookrightarrow G L_{n}$ over $\mathbb{Z}_{p}$, which is often provided by definition for most groups $G$ that arise in practice.
} 
some $n \gg 1$, and $\varphi$-unipotent ${ }^{16}$ if $\mathfrak{M}^{\vee}$ is $\varphi$-nilpotent (i.e., if $\psi_{\mathfrak{M}}^{n}(\mathfrak{M}) \subset \mathfrak{m}_{\mathfrak{S}} \cdot\left(\varphi^{n}\right)^{*} \mathfrak{M}$ for some $n \gg 1$ ).

The following lemma asserts the existence of the linear algebra counterpart of connected-étale and multiplicative-unipotent sequences:

Lemma 5.2. For any $\mathfrak{M} \in \mathrm{BT}_{/ \mathfrak{S}}^{\varphi}$, there exist functorial short exact sequences

$$
\begin{aligned}
& 0 \rightarrow \mathfrak{M}^{\text {ét }} \rightarrow \mathfrak{M} \rightarrow \mathfrak{M}^{c} \rightarrow 0, \\
& 0 \rightarrow \mathfrak{M}^{u} \rightarrow \mathfrak{M} \rightarrow \mathfrak{M}^{m} \rightarrow 0,
\end{aligned}
$$

where $\mathfrak{M}^{\text {ét }}, \mathfrak{M}^{u} \in \mathrm{BT}_{/ \mathfrak{S}}^{\varphi}$ are respectively maximal among étale and $\varphi$-unipotent submodules of $\mathfrak{M}$, and $\mathfrak{M}^{c}, \mathfrak{M}^{m} \in \mathrm{BT}_{/ \mathfrak{S}}^{\varphi}$ are, respectively, maximal among $\varphi$-nilpotent and multiplicative-type quotients of $\mathfrak{M}$. Furthermore, we have $\left(\mathfrak{M}^{\text {ét }}\right)^{\vee}=\left(\mathfrak{M}^{\vee}\right)^{m}$ and $\left(\mathfrak{M}^{c}\right)^{\vee}=\left(\mathfrak{M}^{\vee}\right)^{u}$. The same statement holds for $\mathfrak{M} \in(\operatorname{Mod} / \mathfrak{S})^{\leqslant 1}$.

Proof. When $\mathfrak{M} \in \mathrm{BT}_{/ \mathfrak{S}}^{\varphi}$, the lemma directly follows from [14, Proposition 1.2.11] applied to $\mathfrak{M} /\left(p^{n}\right)$ (which is free over $\mathfrak{S} \otimes_{\mathbb{Z}_{p}} \mathbb{Z}_{p} /\left(p^{n}\right)$ ). When $\mathfrak{M} \in(\operatorname{Mod} / \mathfrak{S})^{\leqslant 1}$, choose $\mathfrak{M} \cong \widetilde{\mathfrak{M}} / \widetilde{\mathfrak{M}^{\prime}}$ with $\widetilde{\mathfrak{M}}, \widetilde{\mathfrak{M}^{\prime}} \in \mathrm{BT}_{/ \mathfrak{S}}^{\varphi}$ and one can check that the sequences induced from equations (5.2.1) and (5.2.2) for $\widetilde{\mathfrak{M}}$ work.

Proposition 5.3. For a $p$-divisible group $G$ over $\mathscr{O}_{K}$, put $\mathfrak{M}_{G}:=\mathfrak{M}^{*}\left(T_{p}(G)\right)$. Then the functor $\mathfrak{M}^{*} \circ T_{p}$ takes the connected-étale and multiplicative-unipotent sequences for $G$ to the exact sequences (5.2.1) and (5.2.2) for $\mathfrak{M}_{G}$, respectively.

We will show later (Section 5.7) that the functor $\mathfrak{M}^{*} \circ T_{p}$ takes any exact sequence of $p$-divisible groups to an exact sequence in $\mathrm{BT}_{/ \mathfrak{S}}^{\varphi}$ (not just connected-étale sequences), and its proof uses the compatibility with crystalline Dieudonné theory whose proof uses Proposition 5.3.

We first need the following lemma that shows an analog to the fact that any étale group scheme over $\mathscr{O}_{\widehat{K}}$ ur is constant.

Lemma 5.3.1. Assume that the residue field $k$ of $\mathscr{O}_{K}$ is algebraically closed. Then $\mathfrak{M} \in \mathrm{BT}_{/ \mathfrak{S}}^{\varphi}$ is étale if and only if $\mathfrak{M}$ is isomorphic to the product of copies of $(\mathfrak{S}, \varphi)$. Also, $\mathfrak{M} \in \mathrm{BT}_{/ \mathfrak{S}}^{\varphi}$ is of multiplicative type if and only if $\mathfrak{M}$ is isomorphic to the product of copies of $(\mathfrak{S}, E(u) \varphi)$.

Proof. Since the two assertions are Cartier dual to each other (cf. Section 5.1), it is enough to prove the étale case. The "if" direction is obvious, so we focus on the "only if" direction. Assume that $\mathfrak{M} \in \mathrm{BT}_{/ \mathfrak{S}}^{\varphi}$ is étale, and we fix a $\mathfrak{S}$-basis $\left\{\mathbf{e}_{1}, \cdots, \mathbf{e}_{n}\right\}$ of $\mathfrak{M}$. Let $A$ denote the matrix representation of $\varphi_{\mathfrak{M}}$ with respect to the chosen basis. If we replace this basis by $\left\{U \mathbf{e}_{i}\right\}$ for some invertible matrix $U$, then $A$ gets replaced by $\varphi(U) A U^{-1}$.

We first show that one can choose an invertible matrix $U_{0}$ over $\mathfrak{S}$ such that $\varphi\left(U_{0}\right) A U_{0}^{-1}$ is congruent to 1 modulo $(u)$; indeed, finding an $n \times n$ invertible matrix $\bar{U}_{0}$ over $W(k)$ such that $\varphi\left(\bar{U}_{0}\right)(A \bmod u) \bar{U}_{0}^{-1}=1$ boils down to solving equations all of whose roots lie in some unramified extension of $W(k)$. So we may

\footnotetext{
${ }^{16}$ Note that $\mathfrak{M}$ being $\varphi$-unipotent does not imply that there exists a unipotent matrix representation of $\varphi_{\mathfrak{M}}$. This notion should be thought of as an analog of unipotent $p$-divisible groups/finite flat group schemes.
} 
assume that $A=1+u B$ for some matrix $B$. Now, by taking $U_{1}=A$, we obtain $A_{1}:=\varphi(A) A A^{-1}=\varphi(A)=1+u^{p} \varphi(B)$. Recursively, we take $U_{i+1}:=A_{i}$ and $A_{i+1}=\varphi\left(U_{i+1}\right) A_{i} U_{i+1}^{-1}$. Since $U_{i+1}:=A_{i} \equiv 1 \bmod u^{p^{i}}$, the infinite product $U:=\prod_{i \geqslant 1} U_{i}$ converges and $\varphi(U) A U^{-1}=1$.

Lemma 5.3.2. Let $\mathfrak{M} \in \mathrm{BT}_{/ \mathfrak{S}}^{\varphi}$, and let $\mathfrak{M}^{\text {ét }}$ be the maximal étale submodule of $\mathfrak{M}$ which exists by Lemma 5.2. Then, $T_{\mathfrak{S}}^{*}\left(\mathfrak{M}^{\text {ét }}\right)$ is the maximal unramified $\mathcal{G}_{K_{\infty}}$-quotient of $T_{\mathfrak{S}}^{*}(\mathfrak{M})$.

Proof. By Lemma 5.3.1, $T_{\mathfrak{S}}^{*}\left(\mathfrak{M}^{\text {ét }}\right)$ is an unramified quotient of $T_{\mathfrak{S}}^{*}(\mathfrak{M})$. To show it is maximal, it is enough to show that $\mathfrak{M}^{\text {ét }} \neq 0$ if $T_{\mathfrak{S}}^{*}(\mathfrak{M})$ has a non-zero unramified quotient. For this, choose $f \in T_{\mathfrak{S}}^{*}(\mathfrak{M})=\operatorname{Hom}_{\mathfrak{S}, \varphi}\left(\mathfrak{M}, \widehat{\mathfrak{S}}^{\text {ur }}\right)$ whose image in the maximal unramified quotient is not zero. Since $\left(\widehat{\mathfrak{S}}^{\text {ur }}\right)^{I_{K}}=W(\bar{k})[[u]]$, it follows that $f^{-1}(W(\bar{k})[[u]])$ is a non-zero étale submodule of $\mathfrak{M}$.

Proof of Proposition 5.3. Since two assertions in the statement are Cartier dual to each other (cf. Section 5.1), it suffices to show that $\mathfrak{M}^{*} \circ T_{p}$ respects connected-étale sequences. Let $\left(G^{\bullet}\right): 0 \rightarrow G^{c} \rightarrow G \rightarrow G^{\text {ét }} \rightarrow 0$ be a connected-étale sequence of $p$-divisible groups, and $\left(\mathfrak{M}^{\bullet}\right): 0 \rightarrow \mathfrak{M}_{G}^{\text {ét }} \rightarrow \mathfrak{M}_{G} \rightarrow \mathfrak{M}_{G}^{c} \rightarrow 0$ denote the "connectedétale sequence" for $\mathfrak{M}_{G}:=\mathfrak{M}^{*}(G)$ as in Lemma 5.2. To prove Proposition 5.3, it suffices to show that $T_{p}\left(G^{\bullet}\right) \cong T_{\mathfrak{S}}^{*}\left(\mathfrak{M}^{\bullet}\right)$ via the natural isomorphism.

By Lemma 5.3.2, $T_{\mathfrak{S}}^{*}\left(\mathfrak{M}_{G}^{t}\right)$ is the maximal unramified $\mathcal{G}_{K_{\infty}}$-quotient of $T_{p}(G)$. Viewing $T_{\mathfrak{S}}^{*}\left(\mathfrak{M}_{G}^{\text {ét }}\right)$ as an unramified $\mathcal{G}_{K}$-representation via $\mathcal{G}_{K_{\infty}} / I_{K_{\infty}} \cong \mathcal{G}_{K} / I_{K}$, we apply the full faithfulness of $\mathcal{G}_{K_{\infty}}$-restriction on crystalline representations [12, Corollary 2.1.14] to conclude that $T_{\mathfrak{S}}^{*}\left(\mathfrak{M}_{G}^{\text {ét }}\right)$ is a maximal unramified $\mathcal{G}_{K^{-}}$quotient of $T_{p}(G)$. This shows that $T_{\mathfrak{S}}^{*}\left(\mathfrak{M}_{G}^{\text {ét }}\right)$ coincides with the quotient $T_{p}\left(G^{\text {ét }}\right)$ of $T_{p}(G)$. Now Proposition 5.3 follows.

The following proposition proves Proposition 4.5 when $\mathfrak{M}$ is either $\varphi$-unipotent or of multiplicative type.

Proposition 5.4. For any $\mathfrak{M} \in \mathrm{BT}_{/ \mathfrak{S}}^{\varphi}$, put $\mathcal{M}:=S \otimes_{\varphi, \mathfrak{S}} \mathfrak{M}$.

(1) Assume that $\mathfrak{M}$ is $\varphi$-unipotent, then the $\mathcal{G}_{K_{\infty}}-$ map $\imath: T_{\mathfrak{S}}^{*}(\mathfrak{M}) \hookrightarrow T_{\mathrm{st}}^{*}(\mathcal{M})$ defined in equation (3.7.1) is an isomorphism.

(2) Assume that $\mathfrak{M}$ is of multiplicative type. If $p>2$ then the $\mathcal{G}_{K_{\infty}}-$ map $\imath$ : $T_{\mathfrak{S}}^{*}(\mathfrak{M}) \hookrightarrow T_{\mathrm{st}}^{*}(\mathcal{M})$ defined in equation $(3.7 .1)$ is an isomorphism. If $p=2$, then the image of $\imath$ is precisely $2 \cdot T_{\mathrm{st}}^{*}(\mathcal{M})$.

Proof. The $\varphi$-unipotent case is exactly [13, Proposition 1.2.7], so it is left to handle the multiplicative-type case. The injectivity of $\imath$ is clear. We will prove the proposition by looking at $(\iota \bmod p)$ and $(\iota \bmod 4)$ when $p=2$.

For the proof, we may replace $K$ by $\widehat{K}^{\text {ur }}$ and $\mathfrak{M}$ by $W(\bar{k})[[u]] \otimes_{\mathfrak{S}} \mathfrak{M}$; indeed, by naturally identifying $V=V_{\text {cris }}^{*}(D) \cong V_{\text {cris }}^{*}\left(W(\bar{k}) \otimes_{W(k)} D\right)$ as $I_{K}$-representations, we do not change the relevant $\mathbb{Z}_{p}$-lattices in $V$ and the map $\imath$. Now, by Lemma 5.3.1 it is enough to prove the proposition for $\mathfrak{M}=(\mathfrak{S}, E(u) \varphi)$. The following claim concludes the proof of Proposition 5.4(2).

Claim 5.4.1. Assume that $k=\bar{k}$ and let $\mathfrak{M}=(\mathfrak{S}, E(u) \varphi)$. If $p>2$ then $(\imath \bmod p)$ is an isomorphism. If $p=2$ then $(\imath \bmod 2)$ is the zero map and $(\imath \bmod 4)$ is non-zero. 
Let $\alpha \in W(k)^{\times}$be such that $\alpha u^{e} \equiv E(u) \bmod p$, and choose $\beta \in W(k)^{\times}$so that $\beta^{p-1} \equiv \alpha^{-1} \bmod p$. Then, for any $f \in \operatorname{Hom}_{\mathfrak{S}, \varphi}\left(\mathfrak{M} /(p), \widehat{\mathfrak{S}}^{\text {ur }} /(p)\right)$ we have

$$
(f(\beta))^{p}=\beta^{p}(f(1))^{p}=u^{e} f(\beta) .
$$

If $f$ is non-zero then we have $f(\beta)^{p-1}=u^{e}$. By Lemma 5.4.2, on the other hand, $\varphi: \widehat{\mathfrak{S}}^{\mathrm{ur}} /(p) \rightarrow A_{\text {cris }} /(p)$ takes $f(\beta)$ to 0 if and only if $p=2$. This proves the claim on $\imath \bmod p$.

Now, assume that $p=2$ and choose a $\mathbb{Z}_{2}$-basis $f \in \operatorname{Hom}_{\mathfrak{S}, \varphi}\left(\mathfrak{M}, \widehat{\mathfrak{S}}^{\text {ur }}\right)$. Since $f(\beta) \equiv u^{e} \bmod 2$, we can find $g \in \widehat{\mathfrak{S}}^{\text {ur }}$ such that $f(\beta)=u^{e}+2 g$. Similarly, we can write $\alpha^{-1} E(u)=u^{e}+2 c(u)$ for some $c(u) \in \mathfrak{S}$ of degree $<e$. Then we have

$$
\varphi(f(\beta)) \equiv \alpha^{-1} E(u) f(\beta) \equiv u^{2 e}+2 u^{e}(c(u)+g) \bmod 4 .
$$

Put $\tilde{f}:=\imath(f) \in \operatorname{Hom}_{S, \varphi_{1}, \operatorname{Fil}^{1}}\left(\mathcal{M}, A_{\text {cris }}\right)$. Then by definition we have

$$
\widetilde{f}(\beta) \equiv u^{2 e}+2 u^{e}(c(u)+g) \equiv 2\left(\frac{u^{2 e}}{2 !}+u^{e} c(u)+u^{e} g\right) \bmod 4 .
$$

Since $u^{2 e} / 2 !+u^{e} c(u)+u^{e} g \not \equiv 0 \bmod 2$ (because $u^{2 e} / 2 ! \notin \mathfrak{S}^{\mathrm{ur}} /(2)$ and $u^{e} c(u)+u^{e} g \in$ $\left.\mathfrak{S}^{\mathrm{ur}} /(2)\right)$, we have $\tilde{f}(\beta) \not \equiv 0 \bmod 4$.

Lemma 5.4.2. The kernel of the map $\varphi: \widehat{\mathfrak{S}}^{\mathrm{ur}} /(p) \rightarrow A_{\mathrm{cris}} /(p)$ is generated by $u^{e}$ where $e=\operatorname{deg} E(u)$.

Proof. Recall that $R$ is isomorphic to the valuation ring of the completion of $k((\underline{\pi}))^{\text {alg }}$, and the subring $\widehat{\mathfrak{S}}^{\mathrm{ur}} /(p) \subset R$ is isomorphic to the valuation ring of $k((u))^{\mathrm{sep}}$ (cf. Section 2.2). Also one can directly check that $A_{\text {cris }} /(p)$ is the divided power envelop of $R$ with respect to the kernel of the 0th projection $\bar{\theta}: R \rightarrow \mathscr{O}_{\bar{K}} /(p) .{ }^{17}$ Since $\operatorname{ker}(\bar{\theta})$ is principally generated by $\underline{\pi}^{e}[28$, Proposition 4.3.7], the kernel of the natural map $R \rightarrow A_{\text {cris }}$ is principally generated by $\underline{\pi}^{p e}$. Now the lemma follows.

The proposition below completes the proof of Proposition 4.5, hence the proof of Theorem 4.1:

Proposition 5.5. For any $\mathfrak{M} \in \mathrm{BT}_{/ \mathfrak{S}}^{\varphi}$ and $\mathcal{M}:=S \otimes_{\varphi, \mathfrak{S}} \mathfrak{M}$, the image of the map $\imath: T_{\mathfrak{S}}^{*}(\mathfrak{M}) \rightarrow T_{\mathrm{st}}^{*}(\mathcal{M})$ is $\mathcal{G}_{K}$-stable, where $\imath$ is defined in equation (3.7.1). If $p>2$ then $\imath$ is an isomorphism, and if $p=2$ then the cokernel of $\imath$ is killed by $p=2$.

Note that a simpler proof when $p>2$ already appeared in the proof of $[12$, Theorem 2.2.7].

Proof. From Proposition 5.4 and the exactness results (Lemmas 3.3 .3 and 3.6), it easily follows that $\imath$ is an isomorphism when $p>2$, and when $p=2$ we have

$$
2 T_{\mathrm{st}}^{*}(\mathcal{M}) \subseteq T_{\mathfrak{S}}^{*}(\mathfrak{M}) \subseteq T_{\mathrm{st}}^{*}(\mathcal{M})
$$

To show that $T_{\mathfrak{S}}^{*}(\mathfrak{M})$ is $\mathcal{G}_{K}$-stable in $T_{\mathrm{st}}^{*}(\mathcal{M})$, it suffices to show that the image of $T_{\mathfrak{S}}^{*}(\mathfrak{M})$ in $T_{\mathrm{st}}^{*}(\mathcal{M}) / 2 T_{\mathrm{st}}^{*}(\mathcal{M})$ is $\mathcal{G}_{K}$-stable; i.e., for any $f \in T_{\mathfrak{S}}^{*}(\mathfrak{M})$ and $g \in \mathcal{G}_{K}$, there

${ }^{17}$ This follows from the universal property of divided power envelop; cf. [2, Remark 3.20(8)]. 
exists $f^{\prime} \in T_{\mathfrak{S}}^{*}(\mathfrak{M})$ such that

$$
g \cdot \imath(f) \equiv \imath\left(f^{\prime}\right) \bmod 2 T_{\mathrm{st}}^{*}(\mathcal{M})
$$

where $g \cdot \imath(f)$ is as defined in equations (3.5.2a) and (3.5.2b).

Recall that we have the short exact sequence $0 \rightarrow \mathfrak{M}^{u} \rightarrow \mathfrak{M} \rightarrow \mathfrak{M}^{m} \rightarrow 0$ defined in equation (5.2.2), which stays exact after applying $S \otimes_{\varphi, \mathfrak{S}}(\cdot)$ by Lemma 3.3.3. We set $\mathcal{M}^{u}:=S \otimes_{\varphi, \mathfrak{S}} \mathfrak{M}^{u}$ and $\mathcal{M}^{m}:=S \otimes_{\varphi, \mathfrak{S}} \mathfrak{M}^{m}$. From Proposition 5.4(1), there exists $f^{\prime} \in T_{\mathfrak{S}}^{*}(\mathfrak{M})$ such that $\left.(g \cdot \imath(f))\right|_{\mathcal{M}^{u}}=\left.\imath\left(f^{\prime}\right)\right|_{\mathcal{M}^{u}}$. So the element $\delta:=g \cdot \imath(f)-\imath\left(f^{\prime}\right)$ lies in $T_{\mathrm{st}}^{*}\left(\mathcal{M}^{m}\right)$. Now it suffices to show that $\delta \in 2 T_{\mathrm{st}}^{*}\left(\mathcal{M}^{m}\right)$; i.e., $\delta(1 \otimes y) \in 2 A_{\text {cris }}$ for any $y \in \mathfrak{M}^{m}$.

Pick $t \in A_{\text {cris }}$ as in Theorem 3.4.4. Since $\mathfrak{M}^{m}$ is multiplicative, we have

$$
\delta\left(\mathcal{M}^{m}\right) \subset(W(\bar{k}) \cdot S) \cdot(t / 2) \subset W(R) \cdot(t / 2) .
$$

It follows from Theorem 3.4.4 that $W(R) \cdot \frac{t}{2} \cap\left(W(R)+2 A_{\text {cris }}\right) \subset 2 A_{\text {cris }}$. (To see this, assume that $a \cdot \frac{t}{2} \in W(R)+2 A_{\text {cris }}$ for some $a \in W(R)$. Then one can find $b, c_{n} \in W(R)$ such that $a \cdot \frac{t}{2}=b+2 \sum_{n=1}^{\infty} c_{n} \gamma_{n}\left(\frac{t}{2}\right)$ in $A_{\text {cris. }}$. By Theorem 3.4.4, $b+\left(-a+2 c_{1}\right) \frac{T}{2}+2 \sum_{n=2}^{\infty} c_{n} \gamma_{n}\left(\frac{T}{2}\right) \in \mathfrak{a}_{\text {cris }}$ must be divisible by $[\underline{\epsilon}]-\sum_{n=0}^{\infty} \gamma_{n}(T)$. This forces that $a \in(2,[\underline{\epsilon}]-1) W(R)$, but since $[\underline{\epsilon}]-1=-\sum_{i=1}^{\infty} 2^{n} \gamma_{n}\left(\frac{t}{2}\right) \in 2 A_{\text {cris }}$ we conclude that $a \in 2 A_{\text {cris }}$.) In particular, we are reduced to showing that $\delta(1 \otimes y) \in$ $W(R)+2 A_{\text {cris }}$ for any $y \in \mathfrak{M}^{m}$. Now, recall that $\imath\left(f^{\prime}\right)(1 \otimes x) \in \varphi\left(\widehat{\mathfrak{S}}^{\text {ur }}\right)$ for any $x \in \mathfrak{M}$ by construction, so it suffices to show that

$$
(g \cdot \imath(f))(1 \otimes x) \in W(R)+2 A_{\text {cris }}
$$

for any $f \in T_{\mathfrak{S}}^{*}(\mathfrak{M}), x \in \mathfrak{M}$, and $g \in \mathcal{G}_{K}$.

With the notation as above, recall from equations (3.5.2a) and (3.5.2b) that

$$
\begin{aligned}
(g \cdot \imath(f))(1 \otimes x) & =g\left(\sum_{i=0}^{\infty} \gamma_{i}\left(-t_{g}\right)\left(\imath(f) \circ N_{\mathcal{M}}^{i}\right)(1 \otimes x)\right) \\
& =g(\imath(f)(1 \otimes x))+g\left(\sum_{i=1}^{\infty} \gamma_{i}\left(-t_{g}\right)\left(\imath(f) \circ N_{\mathcal{M}}^{i}\right)(1 \otimes x)\right),
\end{aligned}
$$

where $\gamma_{i}$ is the standard $i$ th divided power. Inspecting the second row of (5.5.2), the first term is in $W(R)$ by construction, and the second term is in $2 A_{\text {cris }}$ as 2 divides $\gamma_{i}\left(t_{g}\right)$ for any $g \in \mathcal{G}_{K}$ and $i>0$. This shows that equation (5.5.1) holds, hence the proposition.

5.6. Comparison with Dieudonné crystals: proof of Proposition 4.2(1). ${ }^{18}$ Let $G$ be a $p$-divisible group over $\mathscr{O}_{K}, D$ the associated filtered $\varphi$-module, and $\mathbb{D}^{*}(G)$ the contravariantly associated Dieudonné crystal. The $S$-module $\mathbb{D}^{*}(G)(S)$ can be naturally viewed as a strongly divisible $S$-lattice in $S \otimes_{W(k)} D$. (See, for example, $[12,($ A.1)-(A.2)] for more details.) One can actually recover the Dieudonné crystal $\mathbb{D}^{*}(G)$ from the strongly divisible $S$-module $\mathbb{D}^{*}(G)(S) .{ }^{19}$

Let $\mathfrak{M}_{G}:=\mathfrak{M}^{*}\left(T_{p}(G)\right)$ and we associated a strongly divisible $S$-lattice $S \otimes_{\varphi, \mathfrak{S}} \mathfrak{M}_{G}$. The goal of this section is to prove the following lemma.

\footnotetext{
${ }^{18}$ The author thanks T. Liu for providing his idea to improve the original argument.

${ }^{19}$ This can be done by applying [2, Theorem 6.6] to the unique differential operator given by Proposition 3.3.4.
} 
Lemma 5.6.1. With the notation as above, we have $\mathbb{D}^{*}(G)(S)=S \otimes_{\varphi, \mathfrak{S}} \mathfrak{M}_{G}$ as strongly divisible $S$-lattices in $S \otimes_{W(k)} D$.

The starting point of the proof is the following theorem of Faltings, which is the geometric ingredient of the proof.

Theorem 5.6.2 ([9, Section 6 , Theorem 7$])$. There exists a natural $\mathcal{G}_{K}$-equivariant injective map $T_{p}(G) \hookrightarrow T_{\mathrm{st}}^{*}\left(\mathbb{D}^{*}(G)(S)\right)$ which respects the embeddings into $T_{p}(G)\left[\frac{1}{p}\right] \cong$ $V_{\text {cris }}^{*}(D)$. Furthermore, this map is an isomorphism if $p>2$.

To use Faltings's theorem to prove Lemma 5.6.1, we need the following lemma:

Lemma 5.6.3. Let $\mathcal{M}$ and $\mathcal{M}^{\prime}$ be strongly divisible $S$-lattices in $S \otimes_{W(k)} D$, and assume that $\mathcal{M} \cong S \otimes_{\varphi, \mathfrak{S}} \mathfrak{M}$ for some $\mathfrak{M} \in \mathrm{BT}_{/ \mathfrak{S}}^{\varphi}$. If $T_{\mathrm{st}}^{*}(\mathcal{M}) \subseteq T_{\mathrm{st}}^{*}\left(\mathcal{M}^{\prime}\right)$ as $\mathbb{Z}_{p^{-}}$ lattices in $V:=V_{\text {cris }}^{*}(D)$, then we have $\mathcal{M} \supseteq \mathcal{M}^{\prime}$.

Proof. Assume that $\mathcal{M}$ admits a $\mathfrak{S}$-lattice $\mathfrak{M}$ as in the statement. It suffices to show that if $x \in \mathcal{M}\left[\frac{1}{p}\right]$ satisfies $f(x) \in A_{\text {cris }}$ for any $f \in T_{\text {st }}^{*}(\mathcal{M})$, then $m \in \mathcal{M}$. (The lemma follows by applying this to any $x \in \mathcal{M}^{\prime}$.)

Assume to the contrary that there exists $x \in \mathcal{M}\left[\frac{1}{p}\right] \backslash \mathcal{M}$ such that $f(x) \in A_{\text {cris }}$ for any $f \in T_{\text {st }}^{*}(\mathcal{M})$. Let $n$ be the smallest positive integer such that $p^{n} x \in \mathcal{M}$. Then we have $p^{n} x \in \mathcal{M} \backslash p \mathcal{M}$, and $f\left(p^{n} x\right) \in p^{n} A_{\text {cris }}$ for any $f \in T_{\text {st }}^{*}(\mathcal{M})$. But this contradicts to Lemma 5.6.4 below.

Lemma 5.6.4. Suppose $\mathcal{M}=S \otimes_{\varphi, \mathfrak{S}} \mathfrak{M}$ for some $\mathfrak{M} \in \mathrm{BT}_{/ \mathfrak{S}}^{\varphi}$, and let $y \in \mathcal{M}$. If $f(y) \in p A_{\text {cris }}$ for any $f \in T_{\mathrm{st}}^{*}(\mathcal{M})$, then $y \in p \mathcal{M}$.

Proof. By the "multiplicative-unipotent sequence" ( $c f$. equation (5.2.2)), it suffices to handle the case when $\mathfrak{M}$ is either of multiplicative type or $\varphi$-unipotent. The case when $\mathfrak{M}$ is of multiplicative type is straightforward. (For example, one can use Lemma 5.3.1 to reduce to the rank-1 case.) When $\mathfrak{M}$ is $\varphi$-unipotent, the natural map $\iota: T_{\mathfrak{S}}^{*}(\mathfrak{M}) \rightarrow T_{\mathrm{st}}^{*}(\mathcal{M})$ is an isomorphism, so it suffices to prove the same statement for $y \in \mathfrak{M}$ and $f \in T_{\mathfrak{S}}^{*}(\mathfrak{M})$.

Set $\overline{\mathfrak{M}}:=\mathfrak{M} / p \mathfrak{M}$ and $\overline{\mathfrak{N}}:=\bigcap_{\bar{f} \in T_{\mathfrak{S}}^{*}(\overline{\mathfrak{M}})} \operatorname{ker}(\bar{f}) \subseteq \overline{\mathfrak{M}}$. Since $\overline{\mathfrak{N}}$ is a saturated submodule, we have $\overline{\mathfrak{M}} / \overline{\mathfrak{N}} \in(\operatorname{Mod} / \mathfrak{S})^{\leqslant 1}$. (Note that $\mathfrak{S} /(p)=k[[u]]$ is a discrete valuation ring.) By definition of $\overline{\mathfrak{N}}$ we have $T_{\mathfrak{S}}^{*}(\overline{\mathfrak{M}})=T_{\mathfrak{S}}^{*}(\overline{\mathfrak{M}} / \overline{\mathfrak{N}})$, which implies

$$
\operatorname{rank}_{\mathfrak{S} /(p)} \overline{\mathfrak{M}}=\operatorname{dim}_{\mathbb{F}_{p}} T_{\mathfrak{S}}^{*}(\overline{\mathfrak{M}})=\operatorname{dim}_{\mathbb{F}_{p}} T_{\mathfrak{S}}^{*}(\overline{\mathfrak{M}} / \overline{\mathfrak{N}})=\operatorname{rank}_{\mathfrak{S} /(p)} \overline{\mathfrak{M}} / \overline{\mathfrak{N}}
$$

Hence, $\overline{\mathfrak{N}}=0$, which proves the lemma.

Proof of Lemma 5.6.1. Lemma 5.6.1 is easy for étale and multiplicative-type $p$-divisible groups. (Indeed, it essentially boils down to handling $G=\mathbb{G}_{m}\left[p^{\infty}\right]$ or $\mathbb{Q}_{p} / \mathbb{Z}_{p}$, in which case one can explicitly compute and compare both sides of the equality.)

To prove Lemma 5.6.1 it is enough to show an inclusion $S \otimes_{\varphi, \mathfrak{S}} \mathfrak{M}_{G} \supset \mathbb{D}^{*}(G)(S)$ for all $p$-divisible group $G$. Indeed, we can apply this inclusion to the Cartier dual $G^{\vee}$ and obtain the inclusion of the opposite direction. (Note that Cartier duality corresponds to $S$-linear duality on both sides by Section 5.1 and [1, Section 5.3].)

To show this inclusion, it suffices to show $T_{\mathrm{st}}^{*}\left(S \otimes_{\varphi, \mathfrak{S}} \mathfrak{M}_{G}\right) \subset T_{\mathrm{st}}^{*}\left(\mathbb{D}^{*}(G)(S)\right)$ by Lemma 5.6.3. Let $G$ be any $p$-divisible group over $\mathscr{O}_{K}$ and consider the multiplicative- 
unipotent sequence $\left(G^{\bullet}\right): 0 \rightarrow G^{m} \rightarrow G \rightarrow G^{u} \rightarrow 0$; i.e., $G^{m}$ is of multiplicative type and $\left(G^{u}\right)^{\vee}$ is connected. By Proposition 5.3, the sequence $\mathfrak{M}_{G} \bullet:=\mathfrak{M}^{*}\left(T_{p}\left(G^{\bullet}\right)\right)$ is short exact and coincides with the "multiplicative-unipotent sequence" ( $c f$. equation (5.2.2)) for $\mathfrak{M}_{G}$.

We already observed $T_{\mathrm{st}}^{*}\left(S \otimes_{\varphi, \mathfrak{S}} \mathfrak{M}_{G^{m}}\right)=T_{\mathrm{st}}^{*}\left(\mathbb{D}^{*}\left(G^{m}\right)(S)\right)$. Applying Proposition 5.4(1) and Theorem 5.6.2, we obtain that $T_{\mathrm{st}}^{*}\left(S \otimes_{\varphi, \mathfrak{S}} \mathfrak{M}_{G^{u}}\right)=T_{p}\left(G^{u}\right)$ is contained in $T_{\mathrm{st}}^{*}\left(\mathbb{D}^{*}\left(G^{u}\right)(S)\right)$ as $\mathbb{Z}_{p}$-lattices in $V_{p}\left(G^{u}\right)$. Using the exactness of $T_{\mathrm{st}}^{*}$ (Lemma 3.6), one easily obtains the desired inclusion.

5.7. Exactness. We now give a proof of Proposition 4.2(2) and Corollary 4.3, using compatibility with crystalline Dieudonné theory (Proposition 4.2(1)) proved in Section 5.6.

The following lemma proves Proposition 4.2(2)

Lemma 5.7.1. Let $\left(G^{\bullet}\right): 0 \rightarrow G^{\prime} \rightarrow G \rightarrow G^{\prime \prime} \rightarrow 0$ be a short exact sequence of p-divisible groups over $\mathscr{O}_{K}$, and put $\left(\mathfrak{M}^{\bullet}\right):=\mathfrak{M}^{*}\left(G^{\bullet}\right)$.

(1) The sequence $T_{p}\left(G^{\bullet}\right)$ of Tate modules is short exact if and only if $\mathscr{O}_{\mathcal{E}} \otimes_{\mathfrak{S}}\left(\mathfrak{M}^{\bullet}\right)$ is short exact.

(2) If $\left(G^{\bullet}\right)$ is an exact sequence of $p$-divisible groups over $\mathscr{O}_{K}$, then $\left(\mathfrak{M}^{\bullet}\right)$ is an exact sequence.

Proof. By definition we have natural $\mathcal{G}_{K_{\infty}}$-isomorphisms $T_{p}\left(G^{\bullet}\right) \cong T_{\mathfrak{S}}^{*}\left(\mathfrak{M}^{\bullet}\right)$ and the latter is precisely the sequence of étale $\varphi$-modules associated to $T_{p}\left(G^{\bullet}\right)$ by [10, Section B.1.8.3]. Now the statement (1) directly follows from the exactness property of étale $\varphi$-modules. See [10, Section A.1.2] for more details.

Assuming that $\left(G^{\bullet}\right)$ is an exact sequence of $p$-divisible groups over $\mathscr{O}_{K}$, the left exactness of $\left(\mathfrak{M}^{\bullet}\right)$ follows from (2) since the natural map $\mathfrak{M} \rightarrow \mathscr{O}_{\mathcal{E}} \otimes_{\mathfrak{S}} \mathfrak{M}$ is injective for any $\mathfrak{M} \in \mathrm{BT}_{/ \mathfrak{S}}^{\varphi}$. By Nakayama lemma the right exactness could be checked $\bmod u$, and by the compatibility with crystalline Dieudonné theory the sequence $\left(\mathfrak{M}^{\bullet}\right) \otimes_{\mathfrak{S}, \varphi} \mathfrak{S} /(u)$ is isomorphic to the sequence $D^{*}\left(\left(G^{\bullet}\right)_{k}\right)$, where $D^{*}\left(G_{k}\right)$ denote the contravariant Dieudonné module for a $p$-divisible group $G_{k}$ over $k{ }^{20}$ This shows the desired exactness.

Since we have proved Theorem 4.1 and Proposition 4.2, Corollary 4.3 also follows possibly except the exactness assertion (as remarked in the proof of Corollary 4.3). We now show a slightly stronger assertion than the exactness asserted in Corollary 4.3, which can be useful in practice.

Consider a (not necessarily exact) sequence $\left(G^{\bullet}\right): 0 \rightarrow G^{\prime} \rightarrow G \rightarrow G^{\prime \prime} \rightarrow 0$, where $G, G^{\prime}$, and $G^{\prime \prime}$ are either $p$-power order finite flat group schemes or $p$-divisible groups over $\mathscr{O}_{K}$. (From now on, all finite flat group schemes are assumed to be of $p$-power order.) Let $\left(\mathfrak{M}^{\bullet}\right): 0 \rightarrow \mathfrak{M}^{\prime \prime} \rightarrow \mathfrak{M} \rightarrow \mathfrak{M}^{\prime} \rightarrow 0$ denote the (not necessarily exact) sequence of $(\varphi, \mathfrak{S})$-modules corresponding to $\left(G^{\bullet}\right)$. If $G^{\prime}$ is a finite flat group scheme and $G$ is a $p$-divisible group then we define the map $\mathfrak{M} \rightarrow \mathfrak{M}^{\prime}$ as follows: we choose a sufficiently large $n$ so that $G\left[p^{n}\right]$ contains the image of $G^{\prime}$, and consider $\mathfrak{M} \rightarrow \mathfrak{M} /\left(p^{n}\right) \rightarrow \mathfrak{M}^{\prime}$ where the second map is induced from the map $G^{\prime} \rightarrow G\left[p^{n}\right]$ which factors $G^{\prime} \rightarrow G$. Clearly the map $\mathfrak{M} \rightarrow \mathfrak{M}^{\prime}$ is independent of the choice of $n$.

\footnotetext{
${ }^{20}$ For any $p$-divisible group $G$ over $\mathscr{O}_{K}$, we have $\mathbb{D}^{*}(G)(S) \otimes_{S} W(k) \cong D^{*}\left(G_{k}\right)$, hence the desired isomorphism follows from Proposition 4.2(1).
} 
Proposition 5.7.2. We use the same notation as above.

(1) The sequence $\left(G^{\bullet}\right)_{K}$ of the generic fibers is exact if and only if $\mathscr{O}_{\mathcal{E}} \otimes_{\mathfrak{S}}\left(\mathfrak{M}^{\bullet}\right)$ is exact.

(2) The sequence $\left(G^{\bullet}\right)$ is exact if and only if $\left(\mathfrak{M}^{\bullet}\right)$ is exact.

Proof. The proof of Lemma 5.7.1(1) can easily be adapted to prove (1) using the natural $\mathcal{G}_{K_{\infty}}$-isomorphism $T_{\mathfrak{S}}^{*}\left(\mathfrak{M}_{H}\right) \cong H(\bar{K})$ when $H$ is a finite flat group scheme. (When $G^{\prime}$ is a finite flat group scheme and $G$ is a $p$-divisible group, we need the exact sequence (2.6.3).)

The "only if" direction of (2) is already proved except when $G^{\prime}, G$ and $G^{\prime \prime}$ are finite flat group schemes. This case can be handled via a similar argument to the proof of Lemma 5.7.1(2); the left exactness can be deduced from (1) and the injectivity of maps $\left(\mathfrak{M}^{\bullet}\right) \hookrightarrow \mathscr{O}_{\mathcal{E}} \otimes_{\mathfrak{S}}\left(\mathfrak{M}^{\bullet}\right)$, and the right exactness can be checked by reducing $\left(\mathfrak{M}^{\bullet}\right)$ modulo $(u)$ and comparing it with the sequence of contravariant Dieudonné modules for the special fibers $\left(G^{\bullet}\right)_{k}$.

The proof of the "if" direction can be reduced to the case when $G^{\prime}, G$ and $G^{\prime \prime}$ are finite flat group schemes, which follows from the claim below:

Claim A. If $\mathfrak{i}: \mathfrak{M} \rightarrow \mathfrak{M}^{\prime}$ is surjective, then $i: G^{\prime} \rightarrow G$ is a closed immersion.

Granting this claim, we have $\mathfrak{M}_{G / G^{\prime}}=\operatorname{ker}(\mathfrak{i})=\mathfrak{M}^{\prime \prime}$ as submodules of $\mathfrak{M}$, so the natural map $G / G^{\prime} \rightarrow G^{\prime \prime}$ given by the universal property of quotient is an isomorphism. This proves the "if" direction of Proposition 5.7.2(2).

Let us prove Claim A. Let $H \subset G$ be the scheme-theoretic image of $i$, and $\mathfrak{M}_{H}$ the quotient of $\mathfrak{M}$ corresponding to $H$. Since $i$ factors through $H$, it follows that $\mathfrak{i}$ factors through $\mathfrak{M}_{H}$ and we obtain a natural surjective map $\mathfrak{M}_{H} \rightarrow \mathfrak{M}^{\prime}$. This map is an isomorphism if and only if $i$ is a closed immersion. Since both the source and the target have no non-zero $u$-torsion, it suffices to show that the map $\mathscr{O}_{\mathcal{E}} \otimes \mathfrak{i}: \mathscr{O}_{\mathcal{E}} \otimes_{\mathfrak{S}} \mathfrak{M}_{H} \rightarrow$ $\mathscr{O}_{\mathcal{E}} \otimes_{\mathfrak{S}} \mathfrak{M}^{\prime}$ an isomorphism. To show this, observe that the natural map $G_{K}^{\prime} \rightarrow H_{K}$ induced by $i$ on the generic fiber is an isomorphism by Proposition 5.7.2(1). By the theory of étale $\varphi$-modules [10, Section A.1.2] we conclude that the natural surjective map $\mathscr{O}_{\mathcal{E}} \otimes \mathfrak{i}$ is an isomorphism. ${ }^{21}$ This concludes the proof of Claim A (therefore, of Proposition 5.7.2).

\section{Acknowledgments}

The author deeply thanks Brian Conrad, Mark Kisin and Tong Liu for helpful comments. Also he especially thanks the anonymous referee who gave a very careful reading to this paper and provided numerous helpful suggestions, especially on the proof of Proposition 5.5.

\section{References}

[1] P. Berthelot, L. Breen and W. Messing, Théorie de Dieudonné cristalline. II, Vol 930 Lecture Notes in Mathematics, Springer-Verlag, Berlin, 1982.

[2] P. Berthelot and A. Ogus, Notes on crystalline cohomology, Princeton University Press, Princeton, N.J., 1978.

[3] S. Bloch and K. Kato, p-divisible groups and Dieudonné crystals, unpublished, 48 pp.

\footnotetext{
${ }^{21}$ Alternatively, one deduces from Proposition 5.7.2(1) that $\mathscr{O}_{\mathcal{E}} \otimes \mathfrak{i}$ is a surjective map between modules with the same length; hence it is an isomorphism.
} 
[4] C. Breuil, Représentations p-adiques semi-stables et transversalité de Griffiths, Math. Ann. $\mathbf{3 0 7}(2)$ (1997), 191-224.

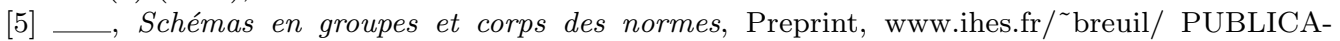
TIONS/groupesnormes.pdf, 1998.

[6] _ Groupes p-divisibles, groupes finis et modules filtrés, Ann. Math. (2) 152(2) (2000), 489-549.

[7] _ Integral p-adic Hodge theory, in 'Algebraic geometry 2000, Azumino (Hotaka)', Vol 36 Adv. Stud. Pure Math. Math. Soc. Japan, Tokyo, 2002, 51-80.

[8] A.J. de Jong, Crystalline Dieudonné module theory via formal and rigid geometry, Inst. Hautes Études Sci. Publ. Math., 82 (1995), 5-96.

[9] G. Faltings, Integral crystalline cohomology over very ramified valuation rings, J. Amer. Math. Soc. 12(1) (1999), 117-144.

[10] J.-M. Fontaine, Représentations p-adiques des corps locaux. I, in 'The Grothendieck Festschrift, Vol. II', Vol 87 of Progr. Math. Birkhäuser Boston, Boston, MA, 1990, 249-309.

[11] _ Le corps des périodes p-adiques, Astérisque, 223 (1994), 59-111. With an appendix by Pierre Colmez, Périodes $p$-adiques (Bures-sur-Yvette, 1988).

[12] M. Kisin, Crystalline representations and F-crystals, in 'Algebraic geometry and number theory', Vol 253 Progr. Math., Birkhäuser Boston, Boston, MA, 2006, 459-496.

[13] _ _ Modularity of 2-adic Barsotti-Tate representations, Invent. Math. 178(3) (2009), 587634.

[14] _ Moduli of finite flat group schemes and modularity, Ann. Math. (2) 170(3) (2009), 10851180.

[15] _ Integral models for Shimura varieties of abelian type, J. Amer. Math. Soc. 23(4) (2010), 967-1012.

[16] S.S. Kudla, Special cycles and derivatives of Eisenstein series, in 'Heegner points and Rankin L-series', Vol 49 of Math. Sci. Res. Inst. Publ. Cambridge University Press, Cambridge, 2004, $243-270$.

[17] R.P. Langlands, Some contemporary problems with origins in the Jugendtraum, in 'Mathematical developments arising from Hilbert problems (Proc. Symp. Pure Math., Vol. XXVIII', Northern Illinois Univ., De Kalb, Ill., 1974), Amer. Math. Soc., Providence, R. I., 1976, 401-418.

[18] E. Lau, Displayed equations for Galois representations, Preprint, http://arxiv.org/ abs/1012.4436v1, 2010.

[19] _ A relation between dieudonne displays and crystalline dieudonne theory, Preprint, http://arxiv.org/abs/1006.2720v1, 2010.

[20] T. Liu, Lattices in filtered $(\varphi, N)$-modules, Preprint, 2008.

[21] _ _ On lattices in semi-stable representations: a proof of a conjecture of Breuil, Compos. Math. 144(1) (2008), 61-88.

[22] —., The correspondence between Barsotti-Tate groups and Kisin modules when $p=2$, Preprint, 2010.

[23] K. Madapusi Sampath, Toroidal compactifications of integral canonical models of Shimura varieties of Hodge type, PhD thesis, University of Chicago, 2011.

[24] B. Mazur and W. Messing, Universal extensions and one dimensional crystalline cohomology, Lecture Notes in Mathematics, Vol. 370, Springer-Verlag, Berlin, 1974.

[25] M. Raynaud, Schémas en groupes de type $(p, \ldots, p)$, Bull. Soc. Math. France 102 (1974), 241-280.

[26] J.T. Tate, p-divisible groups, in 'Proc. Conf. Local Fields (Driebergen, 1966)', Springer, Berlin, 1967, 158-183.

[27] A. Vasiu, Good reductions of shimura varieties of hodge type in arbitrary unramified mixed characteristic, part II, Preprint, http://arxiv.org/abs/0712.1572, 2007.

[28] J.-P. Wintenberger, Le corps des normes de certaines extensions infinies de corps locaux; applications, Ann. Sci. École Norm. Sup. (4) 16(1) (1983), 59-89.

[29] T. Zink, A Dieudonné theory for p-divisible groups, in 'Class field theory - its centenary and prospect (Tokyo, 1998)', Vol 30 Adv. Stud. Pure Math. Math. Soc. Japan, Tokyo, 2001, 139-160.

DPMmS, Centre for Mathematical Sciences, University of Cambridge, Wilberforce Road, CAmbridge CB3 0WB, UK

E-mail address: wk259@dpmms.cam.ac.uk 
